\title{
EXISTENCE, CLASSIFICATION AND STABILITY ANALYSIS OF MULTIPLE-PEAKED SOLUTIONS FOR THE GIERER-MEINHARDT SYSTEM IN $R^{1 *}$
}

\author{
JUNCHENG WEI $^{\dagger}$ AND MATTHIAS WINTER ${ }^{\ddagger}$
}

Abstract. We consider the following Gierer-Meinhardt system in $R^{1}$ :

$$
\begin{cases}A_{t}=\epsilon^{2} A^{\prime \prime}-A+\frac{A^{p}}{H^{q}} & x \in(-1,1), t>0, \\ \tau H_{t}=D H^{\prime \prime}-H+\frac{A^{r}}{H^{s}} & x \in(-1,1), t>0, \\ A^{\prime}(-1)=A^{\prime}(1)=H^{\prime}(-1)=H^{\prime}(1)=0, & \end{cases}
$$

where $(p, q, r, s)$ satisfy

$$
\begin{gathered}
\quad 1<\frac{q r}{(s+1)(p-1)}<+\infty, \quad 1<p<+\infty, \\
\text { and where } \quad \epsilon<<1, \quad 0<D<\infty, \quad \tau \geq 0, \\
D \text { and } \tau \text { are constants which are independent of } \epsilon .
\end{gathered}
$$

We give a rigorous and unified approach to show that the existence and stability of $N$-peaked steadystates can be reduced to computing two matrices in terms of the coefficients $D, N, p, q, r, s$. Moreover, it is shown that $N$-peaked steady-states are generated by exactly two types of peaks, provided their mutual distance is bounded away from zero.

Key words. Stability, Multiple-peaked solutions, Singular perturbations, Turing's instability

AMS subject classifications. Primary 35B40, 35B45; Secondary 35J55, 92C15, 92C40

1. Introduction. Since the work of Turing [26] in 1952, many models have been established and investigated to explore the so-called Turing instability [26]. One of the most famous models in biological pattern formation is the Gierer-Meinhardt system [11], [16], [17], which in one dimension can be stated as follows:

$$
\left\{\begin{array}{l}
A_{t}=\epsilon^{2} \Delta A-A+\frac{A^{p}}{H^{q}} \quad x \in(-1,1), t>0, \\
\tau H_{t}=D \Delta H-H+\frac{A^{r}}{H^{s}} \quad x \in(-1,1), t>0 \\
A^{\prime}( \pm 1, t)=H^{\prime}( \pm 1, t)=0,
\end{array}\right.
$$

where $(p, q, r, s)$ satisfy

$$
\begin{aligned}
& 1<\frac{q r}{(s+1)(p-1)}<+\infty, \quad 1<p<+\infty, \\
& \text { and where } \epsilon<<1, \quad 0<D<\infty, \quad \tau \geq 0,
\end{aligned}
$$

$D$ and $\tau$ are constants which are independent of $\epsilon$.

\footnotetext{
*Received October 30, 2007; accepted for publication April 10, 2008.

${ }^{\dagger}$ Department of Mathematics, The Chinese University of Hong Kong, Shatin, Hong Kong (wei@math. cuhk.edu.hk).

${ }^{\ddagger}$ Brunel University, Department of Mathematical Sciences, Uxbridge, UB8 3PH, United Kingdom (matthias.winter@brunel.ac.uk).
} 
Problem (1.1) has been studied by numerous authors. Let us mention several important results which are related to our present paper.

1) (Existence of symmetric $N$-peaked steady-state Solutions)

I. Takagi [25] first established the existence of $N$-peaked steady-state solutions with peaks centered at

$$
x_{j}=-1+\frac{2 j-1}{N}, \quad j=1, \ldots, N,
$$

for $\epsilon<<1, \frac{\epsilon}{\sqrt{D}}<<1$.

Such solutions are symmetric and they are obtained from a single spike by reflection. We call them symmetric $N$-peaked solution since all the peaks have the same heights. Takagi's proof is based on symmetry and the implicit function theorem.

\section{2) (Stability of symmetric $N$-peaked solutions)}

Using matched asymptotic analysis, D. Iron, M. Ward, and J. Wei [15] studied the stability of the symmetric $N$-peaked solutions for $0 \leq \tau<\tau_{0}$ (where $\tau_{0}>0$ is independent of $\epsilon$ ) and the following results are established (formally):

Result A. There exists a sequence of numbers $D_{1}>D_{2}>\ldots>D_{N}>\ldots$ (which has been given explicitly) such that for $\epsilon<<1$ : If $D<D_{N}$, the symmetric $N$-peaked solutions are stable, while for $D>D_{N}$, the symmetric $N$-peaked solutions are unstable.

In the shadow system case $(D=\infty)$ the existence of single- or $N$-peaked solutions is established in $[1,2,3,13,12,19,20,31,32,33]$ and other papers. In the two-dimensional strong coupling case $(D<\infty)$, the existence of 1-peaked solutions is established in [37], and the stability of $N$-peaked solutions is studied in [38,39]. Results similar to Result $A$ are proved.

\section{3) (Existence of asymmetric $N$-peaked solutions)}

By using the same matched asymptotic analysis in [15], M. Ward and the first author in [28] discovered that problem (1.1) has asymmetric $N$-peaked steady-state solutions which bifurcate off the branch of symmetric $N$-peaked solutions at $D=D_{N}$, where $D_{N}$ is given by Result $A$. Such asymmetric solutions are generated by two types of peaks - called type $\mathbf{A}$ and type $\mathbf{B}$, respectively. Type $\mathbf{A}$ and type $\mathbf{B}$ peaks have different heights. They can be arranged in any given order

\section{ABAABBB...ABBBA...B}

to form an $N$-peaked solution. The existence of such solutions is surprising. It shows that the solution structure of (1.1) is much more complicated than one would expect. The stability of such asymmetric $N$-peaked solutions is also studied in [28], through a formal approach. We remark that asymmetric patterns can also be obtained for the Gierer-Meinhardt system on the real line, see [8].

The purpose of of this paper is to provide a rigorous and unified theoretic foundation for the existence and stability of general $N$-peaked (symmetric or asymmetric) solutions. In particular, the results of [15] and [28] are rigorously established. Moreover, we show that if the $N$ peaks are separated, then they are generated by peaks of type $\mathbf{A}$ and type $\mathbf{B}$, respectively. This implies that there are only two kinds of $N$-peaked patterns: 
symmetric $N$-peaked solutions constructed in [25] and asymmetric $N$-peaked patterns constructed in [28].

The existence proof is based on Liapunov-Schmidt reduction. Stability is proved by first separating the problem into the case of large eigenvalues which tend to a nonzero limit and the case of small eigenvalues which tend to zero in the limit $\epsilon \rightarrow 0$. Large eigenvalues are then explored by studying nonlocal eigenvalue problems using results of [35] and employing an idea of Dancer [5]. Small eigenvalues are calculated explicitly by an asymptotic analysis with rigorous error estimates.

A particular feature of the study of small eigenvalues is that one needs to expand the eigenfunction up to the order $O\left(\epsilon^{2}\right)$ term. This step is different from the single interior peak case [35] and the result is given in Lemma 9.4. We remark that a similar expansion is also needed in the study of small eigenvalues for single boundary spike solutions (see [4] and [34]).

We believe that our approach here, combined with the techniques in [15] and [28], can be very useful in the study of other reaction-diffusion systems as well. With our results we solve a conjecture which was raised in [18].

It turns out that in the case of symmetric $N$-peaked solutions for increasing $D$ the first instability always arises from the small eigenvalues in contrast to the multi-pulses on the real line [6], [7], [9], where the first instability arises from the large eigenvalues.

In [14] the spectra of asymmetric solutions are studied near the point at which they bifurcate off a symmetric branch. It is confirmed that all such solutions are unstable in a neighborhood of the bifurcation point and an explicit expression for the leading order terms of the critical eigenvalues is derived.

A similar analysis for the Fitzhugh-Nagumo model has been carried out in [22]. We note also that in [27], H. van der Ploeg used an alternative dynamical systems approach to study the stability of symmetric spikes.

Before we state our main results in Section 2, we introduce some notation. Let $L^{2}(-1,1)$ and $H^{2}(-1,1)$ be the usual Lebesgue and Sobolev spaces. With the variable $w$ we denote the unique solution of the following problem:

$$
\begin{cases}w^{\prime \prime}-w+w^{p}=0 & \text { in } R^{1}, \\ w>0, w(0)=\max _{y \in R} w(y), w(y) \rightarrow 0 & \text { as }|y| \rightarrow \infty\end{cases}
$$

In fact, it is easy to see that $w(y)$ can be written explicitly

$$
w(y)=\left(\frac{p+1}{2}\right)^{\frac{1}{p-1}}\left(\cosh \left(\frac{p-1}{2} y\right)\right)^{-\frac{2}{p-1}} .
$$

Let $\Omega=(-1,1)$ and $G_{D}(x, z)$ be the Green's function of

$$
\left\{\begin{array}{l}
D G_{D}^{\prime \prime}(x, z)-G_{D}(x, z)+\delta_{z}(x)=0 \quad \text { in }(-1,1), \\
G_{D}^{\prime}(-1, z)=G_{D}^{\prime}(1, z)=0 .
\end{array}\right.
$$

We can calculate explicitly

$$
G_{D}(x, z)= \begin{cases}\frac{\theta}{\sinh (2 \theta)} \cosh [\theta(1+x)] \cosh [\theta(1-z)], & -1<x<z \\ \frac{\theta}{\sinh (2 \theta)} \cosh [\theta(1-x)] \cosh [\theta(1+z)], & z<x<1\end{cases}
$$

where

$$
\theta=D^{-1 / 2}
$$


We set

$$
K_{D}(|x-z|)=\frac{1}{2 \sqrt{D}} e^{-\frac{1}{\sqrt{D}}|x-z|}
$$

to be the non-smooth part of $G_{D}(x, z)$ and by $G_{D}=K_{D}-H_{D}$ we define the regular part $H_{D}$ of $G_{D}$. Note that $H_{D}$ is $C^{\infty}$ in both $x$ and $z$.

We use the notation e.s.t to denote an exponentially small term of order $O\left(e^{-d / \epsilon}\right)$ for some $d>0$ in the corresponding norm. By $C$ we denote a generic constant which may change from line to line.

We shall establish the existence and stability of $N$-peaked steady-state solutions to (1.1). The steady-state problem for (1.1) is the following:

$$
\begin{cases}\epsilon^{2} A^{\prime \prime}-A+\frac{A^{p}}{H^{q}}=0 & \text { in }(-1,1), \\ D H^{\prime \prime}-H+\frac{A^{r}}{H^{s}}=0 & \text { in }(-1,1), \\ A(x)>0, H(x)>0, & \text { in }(-1,1), \\ A^{\prime}(-1)=A^{\prime}(1)=H^{\prime}(-1)=H^{\prime}(1)=0 . & \end{cases}
$$

Since the 1-peaked interior solution has been well-understood in [15], [21], [35] we will assume throughout this paper that

$$
N \geq 2 \text {. }
$$

This paper has the following structure: In Section 2 we introduce our three main hypotheses, (H1) - (H3) and state our three main results, Theorem 2.1, Theorem 2.2 and Theorem 2.3. In Section 3 we study the spectra of a few nonlocal eigenvalue problems on the real line. In Section 4-6 we prove the existence of multiple-peaked solutions: In Section 4 we construct suitable approximate solutions, in Section 5 we use the Liapunov-Schmidt method to reduce the existence of solutions to (1.7) to a finite dimensional problem, in Section 6 we solve this finite-dimensional problem. In Section 7 , we completely classify all possible $N$-peaked solutions, provided the $N$ peaks are separated. In Section 8 we study the large eigenvalues of the linearized operator. In Section 9 we study the small eigenvalues of the linearized operator and give a complete description of their asymptotic behavior in Lemma 9.1. Finally, in the Appendix we compute the eigenvalues of the two main matrices explicitly in the case of symmetric $N$-peaked solutions.

Acknowledgements. The work of JW is supported by an Earmarked Grant of RGC of Hong Kong. MW thanks the Department of Mathematics at CUHK for their kind hospitality. We thank Professor M. J. Ward for valuable discussions.

2. Main Results: Existence and Stability. Let $-1<t_{1}^{0}<\cdots<t_{j}^{0}<\cdots<t_{N}^{0}<1$ be $N$ points in $(-1,1)$ and $w$ be the unique solution of $(1.2)$.

Put

$$
\xi_{\epsilon}:=\left(\epsilon \int_{R} w^{r}(z) d z\right)^{\frac{p-1}{(p-1)(s+1)-q r}} .
$$

We introduce several matrices for later use: For $\mathbf{t}=\left(t_{1}, \ldots, t_{N}\right) \in(-1,1)^{N}$ let

$$
\mathcal{G}_{D}(\mathbf{t})=\left(G_{D}\left(t_{i}, t_{j}\right)\right) .
$$


Recall that

$$
G_{D}\left(t_{i}, t_{j}\right)=K_{D}\left(\left|t_{i}-t_{j}\right|\right)-H_{D}\left(t_{i}, t_{j}\right) .
$$

Let us denote $\frac{\partial}{\partial t_{i}}$ as $\nabla_{t_{i}}$. When $i \neq j$, we can define $\nabla_{t_{i}} G\left(t_{i}, t_{j}\right)$ in the classical way. When $i=j, K_{D}\left(\left|t_{i}-t_{j}\right|\right)=K_{D}(0)=\frac{1}{2 \sqrt{D}}$ is a constant and we define

$$
\nabla_{t_{i}} G_{D}\left(t_{i}, t_{i}\right):=-\left.\frac{\partial}{\partial x}\right|_{x=t_{i}} H\left(x, t_{i}\right) .
$$

Similarly, we define

$$
\nabla_{t_{i}} \nabla_{t_{j}} G_{D}\left(t_{i}, t_{j}\right)=\left\{\begin{array}{l}
-\left.\left.\frac{\partial}{\partial x}\right|_{x=t_{i}} \frac{\partial}{\partial y}\right|_{y=t_{i}} H_{D}(x, y) \text { if } i=j, \\
\nabla_{t_{i}} \nabla_{t_{j}} G_{D}\left(t_{i}, t_{j}\right) \text { if } i \neq j .
\end{array}\right.
$$

Now the derivatives of $\mathcal{G}$ are defined as follows:

$$
\nabla \mathcal{G}_{D}(\mathbf{t})=\left(\nabla_{t_{i}} G_{D}\left(t_{i}, t_{j}\right)\right), \quad \nabla^{2} \mathcal{G}_{D}(\mathbf{t})=\left(\nabla_{t_{i}} \nabla_{t_{j}} G_{D}\left(t_{i}, t_{j}\right)\right) .
$$

We now have our first assumption:

(H1) There exists a solution $\left(\hat{\xi}_{1}^{0}, \ldots, \hat{\xi}_{N}^{0}\right)$ of the following equation

$$
\sum_{j=1}^{N} G_{D}\left(t_{i}^{0}, t_{j}^{0}\right)\left(\hat{\xi}_{j}^{0}\right)^{\frac{q r}{p-1}-s}=\hat{\xi}_{i}^{0}, \quad i=1, \ldots, N .
$$

Next we introduce the following matrix

$$
b_{i j}=G_{D}\left(t_{i}^{0}, t_{j}^{0}\right)\left(\hat{\xi}_{j}^{0}\right)^{\frac{q r}{p-1}-s-1}, \quad \mathcal{B}=\left(b_{i j}\right) .
$$

Our second assumption is the following:

(H2) It holds that

$$
\frac{p-1}{q r-s(p-1)} \notin \sigma(\mathcal{B})
$$

where $\sigma(\mathcal{B})$ is the set of eigenvalues of $\mathcal{B}$.

REMARK 2.1. Since the matrix $\mathcal{B}$ is of the form $\mathcal{G}_{D} \mathcal{D}$, where $\mathcal{G}_{D}$ is symmetric and $\mathcal{D}$ is a diagonal matrix, it is easy to see that the eigenvalues of $\mathcal{B}$ are real.

By the assumption (H2) and the implicit function theorem, for $\mathbf{t}=\left(t_{1}, \ldots, t_{N}\right)$ near $\mathbf{t}_{0}=\left(t_{1}^{0}, \ldots, t_{N}^{0}\right)$, there exists a unique solution $\hat{\xi}(\mathbf{t})=\left(\hat{\xi}_{1}(\mathbf{t}), \ldots, \hat{\xi}_{N}(\mathbf{t})\right)$ for the following equation

$$
\sum_{j=1}^{N} G_{D}\left(t_{i}, t_{j}\right) \hat{\xi}_{j}^{\frac{q r}{p-1}-s}=\hat{\xi}_{i}, \quad i=1, \ldots, N
$$

Set

$$
\mathcal{H}(\mathbf{t})=\left(\hat{\xi}_{i}(\mathbf{t}) \delta_{i j}\right)
$$

We define the following vector field:

$$
F(\mathbf{t})=\left(F_{1}(\mathbf{t}), \ldots, F_{N}(\mathbf{t})\right),
$$


where

$$
\begin{gathered}
F_{i}(\mathbf{t})=\sum_{l=1}^{N} \nabla_{t_{i}} G_{D}\left(t_{i}, t_{l}\right) \hat{\xi}_{l}^{\frac{q r}{p-1}-s} \\
=-\nabla_{t_{i}} H_{D}\left(t_{i}, t_{i}\right) \hat{\xi}^{\frac{q r}{p-1}-s}+\sum_{l \neq i} \nabla_{t_{i}} G_{D}\left(t_{i}, t_{l}\right) \hat{\xi}_{l}^{\frac{q r}{p-1}-s}, \quad i=1, \ldots, N .
\end{gathered}
$$

Set

$$
\mathcal{M}(\mathbf{t})=\left(\hat{\xi}_{i}^{-1} \nabla_{t_{j}} F_{i}(\mathbf{t})\right)
$$

Our final assumption concerns the vector field $F(\mathbf{t})$.

(H3) We assume that at $\mathbf{t}_{0}=\left(t_{1}^{0}, \ldots, t_{N}^{0}\right)$ :

$$
\begin{gathered}
F\left(\mathbf{t}_{0}\right)=0, \\
\operatorname{det}\left(\mathcal{M}\left(\mathbf{t}_{0}\right)\right) \neq 0 .
\end{gathered}
$$

Let us now calculate $\mathcal{M}\left(\mathbf{t}^{0}\right)$ : Therefore we first compute the derivatives of $\hat{\xi}$. It is easy to see that $\hat{\xi}(\mathbf{t})$ is $C^{1}$ in $\mathbf{t}$ and from (2) we can calculate:

$$
\nabla_{t_{j}} \hat{\xi}_{i}=\left(\frac{q r}{p-1}-s\right) \sum_{l=1}^{N} G_{D}\left(t_{i}, t_{l}\right) \hat{\xi}_{l}^{\frac{q r}{p-1}-s-1} \nabla_{t_{j}} \hat{\xi}_{l}+\sum_{l=1}^{N} \frac{\partial}{\partial t_{j}}\left(G_{D}\left(t_{i}, t_{l}\right)\right) \hat{\xi}_{l}^{\frac{q r}{p-1}-s}
$$

For $i \neq j$, we have

$$
\nabla_{t_{j}} \hat{\xi}_{i}=\left(\frac{q r}{p-1}-s\right) \sum_{l=1}^{N} G_{D}\left(t_{i}, t_{l}\right) \hat{\xi}_{l}^{\frac{q r}{p-1}-s-1} \nabla_{t_{j}} \hat{\xi}_{l}+\nabla_{t_{j}} G_{D}\left(t_{i}, t_{j}\right) \hat{\xi}_{j}^{\frac{q r}{p-1}-s} .
$$

For $i=j$, we have

$$
\begin{gathered}
\nabla_{t_{j}} \hat{\xi}_{i}=\left(\frac{q r}{p-1}-s\right) \sum_{l=1}^{N} G_{D}\left(t_{i}, t_{l}\right) \hat{\xi}_{l}^{\frac{q r}{p-1}-s-1} \nabla_{t_{i}} \hat{\xi}_{l}+\sum_{l=1}^{N} \frac{\partial}{\partial t_{i}}\left(G_{D}\left(t_{i}, t_{l}\right)\right) \hat{\xi}_{l}^{\frac{q r}{p-1}-s} \\
=\left(\frac{q r}{p-1}-s\right) \sum_{l=1}^{N} G_{D}\left(t_{i}, t_{l}\right) \hat{\xi}_{l}^{\frac{q r}{p-1}-s-1} \nabla_{t_{i}} \hat{\xi}_{l}+\nabla_{t_{i}} G_{D}\left(t_{i}, t_{i}\right) \hat{\xi}_{i}^{\frac{q r}{p-1}-s}+\sum_{l=1}^{N} \nabla_{t_{i}} G_{D}\left(t_{i}, t_{l}\right) \hat{\xi}_{l}^{\frac{q r}{p-1}-s},
\end{gathered}
$$

since $\frac{\partial}{\partial t_{i}} G_{D}\left(t_{i}, t_{i}\right)=2 \nabla_{t_{i}} G_{D}\left(t_{i}, t_{i}\right)$.

Note that

$$
\left(\nabla_{t_{j}} G_{D}\left(t_{i}, t_{j}\right)\right)=\left(\nabla \mathcal{G}_{D}\right)^{T} .
$$

Therefore if we denote the matrix

$$
\nabla \xi=\left(\nabla_{t_{j}} \hat{\xi}_{i}\right)
$$


then we have

$$
\nabla \xi(\mathbf{t})=\left(I-\left(\frac{q r}{p-1}-s\right) \mathcal{G}_{D} \mathcal{H}^{\frac{q r}{p-1}-s-1}\right)^{-1}\left(\nabla \mathcal{G}_{D}\right)^{T} \mathcal{H}^{\frac{q r}{p-1}-s}+O\left(\sum_{j=1}^{N}\left|F_{j}(\mathbf{t})\right|\right) .
$$

Let

$$
\mathcal{Q}=\left(q_{i j}\right)=\left(\left(-\theta^{2} \hat{\xi}_{i}^{1+s-\frac{q r}{p-1}}+\frac{\theta^{3}}{2}\right) \delta_{i j}\right)
$$

We can compute $\mathcal{M}\left(\mathbf{t}^{0}\right)$ by using (2.15): we note for $i \neq j$ :

$$
\nabla_{t_{j}}\left(\sum_{l=1}^{N} \nabla_{t_{i}} G_{D}\left(t_{i}, t_{l}\right)\right) \hat{\xi}_{l}^{\frac{q r}{p-1}-s}=\left(\nabla_{t_{j}} \nabla_{t_{i}} G_{D}\left(t_{i}, t_{j}\right)\right) \hat{\xi}_{j}^{\frac{q r}{p-1}-s}
$$

and for $i=j$

$$
\begin{gathered}
\nabla_{t_{i}}\left(\sum_{l=1}^{N} \nabla_{t_{i}} G_{D}\left(t_{i}, t_{l}\right)\right) \hat{\xi}_{l}^{\frac{q r}{p-1}-s} \\
=\nabla_{t_{i}}\left(\sum_{l=1, \ldots, N, l \neq i} \nabla_{t_{i}} G_{D}\left(t_{i}, t_{l}\right)\right) \hat{\xi}_{l}^{\frac{q r}{p-1}-s}-\left(\nabla_{t_{i}} \nabla_{t_{i}} H_{D}\left(t_{i}, t_{i}\right)\right) \hat{\xi}_{i}^{\frac{q r}{p-1}} \\
=\frac{1}{D} \sum_{l=1}^{N} G_{D}\left(t_{i}, t_{l}\right) \hat{\xi}_{l}^{\frac{q r}{p-1}-s}-\left(\frac{1}{D} K_{D}(0)\right) \hat{\xi}_{i}^{\frac{q r}{p-1}-s}+\nabla_{t_{i}} \nabla_{t_{i}} G_{D}\left(t_{i}, t_{i}\right) \hat{\xi}_{i}^{\frac{q r}{p-1}-s} \\
=\theta^{2} \hat{\xi}_{i}-\frac{\theta^{3}}{2} \hat{\xi}_{i}^{\frac{q r}{p-1}-s}+\nabla_{t_{i}} \nabla_{t_{i}} G_{D}\left(t_{i}, t_{i}\right) \hat{\xi}_{i}^{\frac{q r}{p-1}-s}
\end{gathered}
$$

and hence

$$
\mathcal{M}\left(\mathbf{t}^{0}\right)=\mathcal{H}^{-1}\left(\nabla^{2} \mathcal{G}_{D}-\mathcal{Q}\right) \mathcal{H}^{\frac{q r}{p-1}-s}
$$

$$
+\mathcal{H}^{-1}\left(\frac{q r}{p-1}-s\right) \nabla \mathcal{G}_{D} \mathcal{H}^{\frac{q r}{p-1}-s-1}\left(I-\left(\frac{q r}{p-1}-s\right) \mathcal{G}_{D} \mathcal{H}^{\frac{q r}{p-1}-s-1}\right)^{-1}\left(\nabla \mathcal{G}_{D}\right)^{T} \mathcal{H}^{\frac{q r}{p-1}-s} .
$$

To simplify our notations, we introduce the following matrices

$$
\begin{gathered}
\mathcal{P}_{1}:=\left(I+s \mathcal{G}_{D} \mathcal{H}^{\frac{q r}{p-1}-s-1}\right)^{-1}, \\
\mathcal{P}_{2}:=\left(I-\left(\frac{q r}{p-1}-s\right) \mathcal{G}_{D} \mathcal{H}^{\frac{q r}{p-1}-s-1}\right)^{-1} .
\end{gathered}
$$

Our first result can be stated as follows:

THEOREM 2.1. Assume that assumptions (H1), (H2) and (H3) are satisfied. Then for $\epsilon<<1$, problem (1.7) has an $N$-peaked solution which concentrates at $t_{1}^{\epsilon}, \ldots, t_{N}^{\epsilon}$, or more precisely:

$$
A_{\epsilon}(x) \sim \sum_{j=1}^{N} \xi_{\epsilon}^{\frac{q}{p-1}}\left(\hat{\xi}_{j}{ }^{0}\right)^{\frac{q}{p-1}} w\left(\frac{x-t_{j}^{\epsilon}}{\epsilon}\right)
$$




$$
\begin{gathered}
H_{\epsilon}\left(t_{i}^{\epsilon}\right) \sim \xi_{\epsilon} \hat{\xi}_{i}^{0}, \quad i=1, \ldots, N \\
t_{i}^{\epsilon} \rightarrow t_{i}^{0}, \quad i=1, \ldots, N .
\end{gathered}
$$

REMARK 2.2. In the case of symmetric $N$-peaked solutions, conditions (H2) and (H3) are not needed, as in the construction of solutions one can restrict the function space to the class of symmetric functions (see for example [25]). Note that for small $\epsilon$ (and not only in the limit $\epsilon \rightarrow 0$ ) the peaks are placed equidistantly.

REMARK 2.3. Our results here can be applied to give a rigorous proof for the existence and stability of $N$-peaked solutions consisting of peaks with different heights.

In [28], by using matched asymptotic analysis, Ward and the first author constructed such solutions and studied their stability. We now summarize their main ideas. First (1.7) is solved in a small interval $(-l, l)$ :

$$
\begin{cases}\epsilon^{2} A^{\prime \prime}-A+\frac{A^{p}}{H^{q}}=0 & \text { in }(-l, l), \\ D H^{\prime \prime}-H+\frac{A^{r}}{H^{s}}=0 & \text { in }(-l, l), \\ A(x)>0, H(x)>0 & \text { in }(-l, l), \\ A^{\prime}(-l)=A^{\prime}(l)=H^{\prime}(-l)=H^{\prime}(l)=0 . & \end{cases}
$$

Then the single interior symmetric spike solution is considered which was constructed by I. Takagi [25]. By some simple computations based on (1.4), we have that

$$
H(l) \sim c(D) b\left(\frac{l}{\sqrt{D}}\right),
$$

where $c(D)$ is some positive constant depending on $D$ only and the function $b(z)$ is given by

$$
b(z):=\frac{\tanh ^{\alpha}(z)}{\cosh (z)}, \quad \alpha:=\frac{(p-1)}{q r-(s+1)(p-1)} .
$$

The idea now is that we fix $l$ and try to find another $\bar{l} \neq l$ such that the following holds

$$
b\left(\frac{l}{\sqrt{D}}\right)=b\left(\frac{\bar{l}}{\sqrt{D}}\right), \quad 0<l<\bar{l}<1,
$$

which will imply that $H(l) \sim H(\bar{l})$. This shows that if there exists a solution to (2.26), we may match up $H(l)$ and $H(\bar{l})$. In other words, we may match up solutions of $(2.23)$ in different intervals.

It turns out that for $D$ small, (2.26) is always solvable. Now (2.26) has to be solved along with the following interval constraint:

$$
N_{1} l+N_{2} \bar{l}=1, \quad N_{1}+N_{2}=N .
$$

For a solution $l$ of $(2.26)$ and $(2.27)$ and $j=1, \ldots, N$ we define

$$
l_{j}=l \text { or } l_{j}=\bar{l}
$$


where the number of $j$ 's such that $l_{j}=l$ is $N_{1}$ (and consequently the number of $j$ 's such that $l_{j}=\bar{l}$ is $N_{2}$ ). We call the small spike with $l_{j}=l$ type $\mathbf{A}$ and the large spike with $l_{j}=\bar{l}$ type $\mathbf{B}$.

Then we choose $t_{j}^{0}$ such that

$$
\left|t_{j}^{0}-t_{j+1}^{0}\right|=l_{j}+l_{j-1}, \quad j=0, \ldots, N,
$$

where $t_{0}^{0}=-1, t_{N+1}^{0}=1$.

By using matched asymptotics, we now have $N_{1}$ type $\mathbf{A}$ and $N_{2}$ type $\mathbf{B}$ peaks. This ends our short review of the ideas in [28]. Let us now use Theorem 2.1 to give a rigorous proof of results of [28]. In order to apply Theorem 2.1, we have to check the three assumptions (H1), (H2) and (H3).

To this end, let us set

$$
\hat{\xi}_{j}^{0}=(2 \sqrt{D}) \tanh \left(\theta_{j}\right), \quad j=1, \ldots, N,
$$

where

$$
\theta_{j}=\frac{l_{j}}{\sqrt{D}} .
$$

It is difficult to check (H1) directly. Instead we note that $\mathcal{G}_{D}^{-1}$ is a tridiagonal matrix. (See [15] and [28].) More precisely, we calculate

$$
\mathcal{G}_{D}^{-1}=\left(a_{i j}\right)=2 \sqrt{D}\left(\begin{array}{cccccc}
\gamma_{1} & \beta_{1} & 0 & \ddots & \ddots & 0 \\
\beta_{1} & \gamma_{2} & \beta_{2} & \ddots & \ddots & \\
\ddots & \ddots & \ddots & \ddots & \ddots & \ddots \\
\ddots & \ddots & \beta_{j-1} & \gamma_{j} & \beta_{j} & 0 \\
\ddots & \ddots & \ddots & \ddots & \ddots & \ddots \\
0 & \ddots & \ddots & 0 & \beta_{N-1} & \gamma_{N}
\end{array}\right)
$$

where

$$
\begin{gathered}
\gamma_{1}=\operatorname{coth}\left(\theta_{1}+\theta_{2}\right)+\tanh \left(\theta_{1}\right), \\
\gamma_{j}=\operatorname{coth}\left(\theta_{j-1}+\theta_{j}\right)+\operatorname{coth}\left(\theta_{j}+\theta_{j+1}\right), \quad j=2, \ldots, N-1, \\
\gamma_{N}=\operatorname{coth}\left(\theta_{N-1}+\theta_{N}\right)+\tanh \left(\theta_{N}\right), \\
\beta_{j}=-\operatorname{csch}\left(\theta_{j}+\theta_{j+1}\right), \quad j=1, \ldots, N-1
\end{gathered}
$$

and $\theta_{j}$ was defined in (2.30). Note that

$$
a_{i j}=2 \sqrt{D}\left(\beta_{j} \delta_{i(j-1)}+\gamma_{j} \delta_{i j}+\beta_{j+1} \delta_{i(j+1)}\right) .
$$

Verifying (2.5) amounts to checking the following identity

$$
\sum_{j=1}^{N} a_{i j} \hat{\xi}_{j}^{0}=\left(\hat{\xi}_{i}^{0}\right)^{\frac{q r}{p-1}-s},
$$


which is an easy exercise.

It remains to verify (H2) and (H3).

To this end, we need to know the eigenvalues of $\mathcal{B}$ and $\mathcal{M}$. In the same way as for the matrix $\mathcal{G}_{D}$, one can show that $\mathcal{B}^{-1}$ is a tridiagonal matrix. Even with this piece of information, it is almost impossible to obtain an explicit formula for the eigenvalues. Numerical software for solving eigenvalue problems of large matrices is indispensable. Then (H2) has to be checked explicitly. Numerical computations in [28] do suggest that assumption (H3) is always satisfied.

A natural question is the following: Are all $N$-peaked solutions generated by two types of peaks as the solutions which were constructed in [28]?

Our next theorem gives an affirmative answer. It completely classifies all $N$-peaked solutions, provided that the $N$ peaks are separated.

ThEOREM 2.2. Suppose that for $\epsilon$ sufficiently small, there are solutions $\left(A_{\epsilon}, H_{\epsilon}\right)$ of (1.7) such that

$$
A_{\epsilon}(x) \sim \sum_{j=1}^{N} \xi_{\epsilon}^{\frac{q}{p-1}}\left(\hat{\xi}_{j}^{\epsilon}\right)^{\frac{q}{p-1}} w\left(\frac{x-t_{j}^{\epsilon}}{\epsilon}\right)
$$

and

$$
H_{\epsilon}\left(t_{i}^{\epsilon}\right) \sim \xi_{\epsilon} \hat{\xi}_{i}^{\epsilon}, \quad i=1, \ldots, N
$$

where $\xi_{\epsilon}$ is given by (2.1),

$$
\hat{\xi}_{i}^{\epsilon} \rightarrow \hat{\xi}_{i}^{0}>0, t_{i}^{\epsilon} \rightarrow t_{i}^{0}, t_{i}^{0} \neq t_{j}^{0}, i \neq j, \quad i, j=1, \ldots, N .
$$

Then necessarily, we have

$$
l_{i}:=t_{i}^{0}-t_{i-1}^{0} \in\{l, \bar{l}\}, i=1, \ldots, N,
$$

where $t_{0}^{0}=-1, l$ and $\bar{l}$ satisfy (2.26) and (2.27) with $N_{1}$ being the number of $i$ 's for which $l_{i}=l$ and $N_{2}$ being the number of $i$ 's for which $l_{i}=\bar{l}$ (hence $N_{1}+N_{2}=N$ ).

Theorem 2.2 shows that an $N$-peaked solution must be generated by exactly two types of peaks - type $\mathbf{A}$ with shorter length $l$ and type $\mathbf{B}$ with larger length $\bar{l}$. This shows that the solutions constructed in [28] (through a formal approach) exhaust all possible separated $N$-peaked solutions. In particular, it shows that there are at most $2^{N} N$-peaked solutions. If the assumptions (H1)-(H3) are met, then there are exactly $2^{N} N$-peaked solutions.

Finally, we study the stability of the $N$-peaked solutions constructed in Theorem 2.1.

Theorem 2.3. Let $\left(A_{\epsilon}, H_{\epsilon}\right)$ be the solutions constructed in Theorem 2.1. Assume that $\epsilon<<1$.

(1) (Stability) If

$$
r=2, \quad 1<p<5 \text { or } r=p+1,1<p<+\infty
$$

and furthermore

$$
\left(\frac{q r}{p-1}-s\right) \min _{\sigma \in \sigma(\mathcal{B})} \sigma>1
$$

and

$$
\sigma(\mathcal{M}) \subseteq\{\sigma \mid \operatorname{Re}(\sigma)>0\},
$$


there exists $\tau_{0}>0$ such that $\left(A_{\epsilon}, H_{\epsilon}\right)$ is linearly stable for $0 \leq \tau<\tau_{0}$.

(2) (Instability) If

$$
\left(\frac{q r}{p-1}-s\right) \min _{\sigma \in \sigma(\mathcal{B})} \sigma<1,
$$

there exists $\tau_{0}>0$ such that $\left(A_{\epsilon}, H_{\epsilon}\right)$ is linearly unstable for $0 \leq \tau<\tau_{0}$.

(3) (Instability) If there exists

$$
\sigma \in \sigma(\mathcal{M}), \operatorname{Re}(\sigma)<0,
$$

then $\left(A_{\epsilon}, H_{\epsilon}\right)$ is linearly unstable for all $\tau>0$.

REMARK 2.4. In the original Gierer-Meinhardt model, $(p, q, r, s)=(2,1,2,0)$ or $(p, q, r, s)=(2,4,2,0)$. This means that condition (2.37) is satisfied. In the general case, one has to study a nonlocal eigenvalue problem (Theorem 3.1), which is difficult since the operator is not self-adjoint. See [5], [40] for progress in this direction.

REMARK 2.5. For the stability, we have to assume that $0 \leq \tau<\tau_{0}$ for some $\tau_{0}>0$ which we do not know explicitly. Stability in the case where $\tau$ is large has been investigated in [29] and [30] for symmetric spikes.

For the case of asymmetric spikes, the stability problem with respect to the large eigenvalues remains mainly open. It is expected that there is stability with respect to the large eigenvalues for some range for $D>D_{N}$ if $D$ is sufficiently close to $D_{N}$ and $\tau$ is small enough.

We remark that stability in the case of large $\tau$ for the shadow system has been studied in [5].

REMARK 2.6. By Theorem 2.1 and Theorem 2.3, the existence and stability of $N$-peaked solutions are completely determined by the two matrices $\mathcal{B}$ and $\mathcal{M}$. They are related to the asymptotic behavior of large eigenvalues which tend to a nonzero limit and small eigenvalues which tend to zero as $\epsilon \rightarrow 0$, respectively. The computations of these two matrices are by no means easy. We refer to [15] and [28] for exact computations and numerics. For the reader's convenience, we include in the Appendix A a sketch of the computations of the eigenvalues of the matrices $\mathcal{B}$ and $\mathcal{M}$ in the symmetric $N$-peaked case. Combining the results here and the computations in [15], the stability of symmetric $N$-peaked solutions is completely characterized and the following result is established rigorously.

TheOREM 2.4. Let $\left(A_{\epsilon, N}, H_{\epsilon, N}\right)$ be the symmetric $N$-peaked solutions constructed by I. Takagi [25]. Assume that $\epsilon>>1$.

(a) (Stability) Assume that $0<\tau<\tau_{0}$ for some $\tau_{0}$ small and that

$$
r=2, \quad 1<p<5 \text { or } r=p+1,1<p<+\infty
$$

and

$$
D<D_{N}:=\frac{1}{N^{2}(\log (\sqrt{\alpha}+\sqrt{\alpha+1}))^{2}},
$$

where $\alpha$ is given by (2.25), then the symmetric $N$-peaked solution is linearly stable.

(b) (Instability) If

$$
D>D_{N},
$$

where $D_{N}$ is given by (2.43), then the $N$-peaked solution is linearly unstable for all $\tau>0$.

The proof of Theorem 2.4 is given in Appendix A. 
3. Some preliminaries. In this section, we consider a system of nonlocal linear operators. We first recall

THEOREM 3.1. Consider the following nonlocal eigenvalue problem

$$
\phi^{\prime \prime}-\phi+p w^{p-1} \phi-\gamma(p-1) \frac{\int_{R} w^{r-1} \phi}{\int_{R} w^{r}} w^{p}=\alpha \phi .
$$

(1) (Appendix $E$ of [15].) If $\gamma<1$, then there is a positive eigenvalue to (3.1).

(2) (Theorem 1.4 of [35].) If $\gamma>1$ and (2.37) holds then for any nonzero eigenvalue $\alpha$ of (3.1), we have

$$
\operatorname{Re}(\alpha) \leq-c_{0}<0
$$

(3) If $\gamma \neq 1$ and $\alpha=0$, then $\phi=c_{0} w^{\prime}$ for some constant $c_{0}$.

In our applications to the case when $\tau>0$, we have to deal with the situation when the coefficient $\gamma$ is a function of $\tau \alpha$. Let $\gamma=\gamma(\tau \alpha)$ be a complex function of $\tau \alpha$. Let us suppose that

$$
\gamma(0) \in R, \quad|\gamma(\tau \alpha)| \leq C \text { for } \alpha_{R} \geq 0, \tau \geq 0,
$$

where $C$ is a generic constant independent of $\tau, \alpha$. A simple example of $\sigma(\tau \alpha)$ satisfying $(3.2)$ is

$$
\sigma(\tau \alpha)=\frac{2}{\sqrt{1+\tau \alpha}+1}
$$

where $\sqrt{1+\tau \alpha}$ is the principal branch.

Now we have

Theorem 3.2. Consider the following nonlocal eigenvalue problem

$$
\phi^{\prime \prime}-\phi+p w^{p-1} \phi-\gamma(\tau \alpha)(p-1) \frac{\int_{R} w^{r-1} \phi}{\int_{R} w^{r}} w^{p}=\alpha \phi,
$$

where $\gamma(\tau \alpha)$ satisfies (3.2). Then there is a small number $\tau_{0}>0$ such that for $\tau<\tau_{0}$,

(1) if $\gamma(0)<1$, then there is a positive eigenvalue to (3.1);

(2) if $\gamma(0)>1$ and (2.37) holds, then for any nonzero eigenvalue $\alpha$ of (3.3), we have

$$
\operatorname{Re}(\alpha) \leq-c_{0}<0
$$

Proof. Theorem 3.2 follows from Theorem 3.1 by a perturbation argument. To make sure that the perturbation argument works, we have to show that if $\alpha_{R} \geq 0$ and $0<\tau<1$, then $|\alpha| \leq C$, where $C$ is a generic constant (independent of $\tau$ ). In fact, multiplying (3.3) by $\bar{\phi}$ - the conjugate of $\phi$ - and integrating by parts, we obtain that

$$
\int_{R}\left(\left|\phi^{\prime}\right|^{2}+|\phi|^{2}-p w^{p-1}|\phi|^{2}\right)=-\alpha \int_{R}|\phi|^{2}-\gamma(\tau \alpha)(p-1) \frac{\int_{R} w^{r-1} \phi}{\int_{R} w^{r}} \int_{R} w^{p} \bar{\phi} .
$$

From the imaginary part of (3.4), we obtain that

$$
\left|\alpha_{I}\right| \leq C_{1}|\gamma(\tau \alpha)|,
$$


where $\alpha=\alpha_{R}+\sqrt{-1} \alpha_{I}$ and $C_{1}$ is a positive constant (independent of $\tau$ ). By assumption (3.2), $|\gamma(\tau \alpha)| \leq C$ and so $\left|\alpha_{I}\right| \leq C$. Taking the real part of (3.4) and noting that

$$
\text { 1.h.s. of }(3.4) \geq C \int_{R}|\phi|^{2} \quad \text { for some } C \in R \text {, }
$$

we obtain that $\alpha_{R} \leq C_{2}$, where $C_{2}$ is a positive constant (independent of $\tau>0$ ). Therefore, $|\alpha|$ is uniformly bounded and hence a perturbation argument gives the desired conclusion. $\square$

Next, we consider the following system of linear operators

$$
\begin{gathered}
L \Phi:=\Phi^{\prime \prime}-\Phi+p w^{p-1} \Phi \\
-q r(I+s \mathcal{B})^{-1} \mathcal{B}\left(\int_{R} w^{r-1} \Phi\right)\left(\int_{R} w^{r}\right)^{-1} w^{p},
\end{gathered}
$$

where $\mathcal{B}$ is given by $(2.6)$ and

$$
\Phi=\left(\begin{array}{l}
\phi_{1} \\
\phi_{2} \\
\vdots \\
\phi_{N}
\end{array}\right) \in\left(H^{2}(R)\right)^{N}
$$

Set

$$
L_{0} u:=u^{\prime \prime}-u+p w^{p-1} u, \quad \text { where } u \in H^{2}(R) .
$$

Then using Remark 2.1 the conjugate operator of $L$ under the scalar product in $L^{2}(R)$ is

$$
\begin{gathered}
L^{*} \Psi=\Psi^{\prime \prime}-\Psi+p w^{p-1} \Psi \\
-q r \mathcal{B}^{T}\left(I+s \mathcal{B}^{T}\right)^{-1}\left(\int_{R} w^{p} \Psi\right)\left(\int_{R} w^{r}\right)^{-1} w^{r-1},
\end{gathered}
$$

where

$$
\Psi=\left(\begin{array}{l}
\psi_{1} \\
\psi_{2} \\
\vdots \\
\psi_{N}
\end{array}\right) \in\left(H^{2}(R)\right)^{N}
$$

We obtain the following

LEMma 3.3. Assume that assumption (H2) holds. Then

$$
\operatorname{Ker}(L)=X_{0} \oplus X_{0} \oplus \cdots \oplus X_{0},
$$

where

$$
X_{0}=\operatorname{span}\left\{w^{\prime}(y)\right\}
$$

and

$$
\operatorname{Ker}\left(L^{*}\right)=X_{0} \oplus X_{0} \oplus \cdots \oplus X_{0} .
$$


Proof. Let us first prove (3.8). Suppose

$$
L \Phi=0 .
$$

Let us diagonalize $\mathcal{B}$ such that

$$
P^{-1} \mathcal{B P}=J,
$$

where $P$ is an orthogonal matrix and by Remark $2.1 J$ has diagonal form, i.e.,

$$
J=\left(\begin{array}{cccc}
\sigma_{1} & & & 0 \\
& \sigma_{2} & & \\
& & \ddots & \\
0 & & & \sigma_{N}
\end{array}\right)
$$

with suitable real numbers $\sigma_{j}, \quad j=1,2, \ldots, N$.

Defining

$$
\Phi=P \tilde{\Phi}
$$

we have

$$
\tilde{\Phi}^{\prime \prime}-\tilde{\Phi}+p w^{p-1} \tilde{\Phi}-q r\left(\int_{R} w^{r}\right)^{-1}\left(\int_{R} w^{r-1}(I+s J)^{-1} J \tilde{\Phi}\right) w^{p}=0 .
$$

For $l=1,2, \ldots, N$ we look at the $l$-th equation of system (3.10):

$$
\begin{gathered}
\tilde{\Phi}_{l}^{\prime \prime}-\tilde{\Phi}_{l}+p w^{p-1} \tilde{\Phi}_{l} \\
-q r\left(\int_{R} w^{r}\right)^{-1}\left(\frac{\sigma_{l}}{1+s \sigma_{l}} \int_{R} w^{r-1} \tilde{\Phi}_{l}\right) w^{p}=0 .
\end{gathered}
$$

By Theorem 3.1 (3), the last equation (3.11) tells us that (since by condition (H2) we know $\left.q r \frac{\sigma_{l}}{1+s \sigma_{l}} \neq p-1\right)$

$$
\tilde{\Phi}_{l} \in X_{0}
$$

Continuing in this way for $l=1, \ldots, N$, we have

$$
\tilde{\Phi}_{l} \in X_{0}, l=1, \ldots, N .
$$

(3.8) is thus proved.

To prove (3.9), we proceed in the same way for $L^{*}$.

Using $\sigma(\mathcal{B})=\sigma\left(\mathcal{B}^{T}\right)$ the $l$-th equation of the diagonalized system is as follows:

$$
\begin{gathered}
\tilde{\Psi}_{l}^{\prime \prime}-\tilde{\Psi}_{l}+p w^{p-1} \tilde{\Psi}_{l} \\
-q r\left(\int_{R} w^{r}\right)^{-1} \frac{\sigma_{l}}{1+s \sigma_{l}}\left(\int_{R} w^{p} \tilde{\Psi}_{l}\right) w^{r-1}=0 .
\end{gathered}
$$

Multiplying (3.14) by $w$ and integrating over the real line, we obtain

$$
\left(p-1-q r \frac{\sigma_{l}}{1+s \sigma_{l}}\right) \int_{R} w^{p} \tilde{\Psi}_{l}=0,
$$


which implies that

$$
\int_{R} w^{p} \tilde{\Psi}_{l}=0
$$

since $q r \frac{\sigma_{l}}{1+s \sigma_{l}} \neq p-1$.

Thus all the nonlocal terms vanish and we have

$$
L_{0} \tilde{\Psi}_{l}=0, \quad l=1, \ldots, N .
$$

This implies that $\tilde{\Psi}_{l} \in X_{0}$ for $l=1, \ldots, N$.

As a consequence of Lemma 3.3, we have

LEMma 3.4. The operator

$$
L:\left(H^{2}(R)\right)^{N} \rightarrow\left(L^{2}(R)\right)^{N}
$$

is invertible if it is restricted as follows

$$
L:\left(X_{0} \oplus \cdots \oplus X_{0}\right)^{\perp} \cap\left(H^{2}(R)\right)^{N} \rightarrow\left(X_{0} \oplus \cdots \oplus X_{0}\right)^{\perp} \cap\left(L^{2}(R)\right)^{N} .
$$

Moreover, $L^{-1}$ is bounded.

Proof. This follows from the Fredholm Alternatives Theorem and Lemma 3.3.

Finally, we study the eigenvalue problem for $L$ :

$$
L \Phi=\alpha \Phi .
$$

We have

Lemma 3.5. Assume that all the eigenvalues of $\mathcal{B}$ are real. Then we have:

(1) If $\left(\frac{q r}{p-1}-s\right) \min _{\sigma \in \sigma(\mathcal{B})}>1$, then for any nonzero eigenvalue of (3.16) we must have $\alpha \leq-c_{0}<0$.

(2) If there exists $\sigma \in \sigma(\mathcal{B})$ such that $\left(\frac{q r}{p-1}-s\right) \sigma<1$, then there exists a positive eigenvalue of (3.16).

Proof. Let $(\Phi, \alpha)$ satisfy (3.16). Suppose $\alpha_{R} \geq 0$ and $\alpha \neq 0$. Similar to Lemma 3.3, we diagonalize $(3.16)$

$$
\Phi^{\prime \prime}-\Phi+p w^{p-1} \Phi-q r\left(\int_{R} w^{r}\right)^{-1}\left(\int_{R} w^{r-1}(I+s J)^{-1} J \Phi\right) w^{p}=\alpha \Phi
$$

and the $l$-th equation of system (3.17) becomes

$$
\Phi_{l}^{\prime \prime}-\Phi_{l}+p w^{p-1} \Phi_{l}-q r \frac{\sigma_{l}}{1+s \sigma_{l}}\left(\int_{R} w^{r}\right)^{-1}\left(\int_{R} w^{r-1} \Phi_{l}\right) w^{p}=\alpha \Phi_{l} .
$$

(i) By Theorem 3.1 (1) and the fact that

$$
\frac{q r}{p-1} \frac{\sigma_{l}}{1+s \sigma_{l}}>1
$$

we conclude that

$$
\Phi_{1}=\cdots=\Phi_{N}=0
$$


or

$$
\alpha \leq-c_{0}<0
$$

Since by assumption the eigenfunctions are non-vanishing the second alternative holds. (1) is proved. becomes

(ii) if $\sigma_{l}\left(\frac{q r}{p-1}-s\right)<1$ for some $\sigma_{l} \in \sigma(\mathcal{B})$, then the equation corresponding to $\sigma_{l}$

$$
\Phi_{l}^{\prime \prime}-\Phi_{l}+p w^{p-1} \Phi_{l}-q r \frac{\sigma_{l}}{1+s \sigma_{l}}\left(\int_{R} w^{r}\right)^{-1}\left(\int_{R} w^{r-1} \Phi_{l}\right)=\alpha \Phi_{l} .
$$

By Theorem 3.1 (2), we know that there exists an eigenvalue $\alpha_{0}>0$ and an eigenfunction $\Phi_{0}$ such that

$$
L_{0} \Phi_{0}-q r\left(\int_{R} w^{r}\right)^{-1}\left(\frac{\sigma_{l}}{1+s \sigma_{l}} \int_{R} w^{r-1} \Phi_{0}\right)=\alpha_{0} \Phi_{0} .
$$

Let us take $\Phi_{l}=\Phi_{0}$ and $\Phi_{j}=0$ for $j \neq l$. Then $(\Phi, \alpha)$ satisfy (3.16). (2) is proved.

4. Study of the approximate solutions. Let $-1<t_{1}^{0}<\cdots<t_{j}^{0}<\cdots t_{N}^{0}<1$ be $N$ points satisfying the assumptions $(\mathrm{H} 1)-(\mathrm{H} 3)$. Let $\hat{\xi}^{0}=\left(\hat{\xi}_{1}^{0}, \ldots, \hat{\xi}_{N}^{0}\right)$ be the unique solution of (2.5). Let

$$
\mathbf{t}^{0}=\left(t_{1}^{0}, \ldots, t_{N}^{0}\right)
$$

We now construct an approximate solution to (1.7) which concentrates near these prescribed $N$ points.

Let $-1<t_{1}<\cdots<t_{j}<\cdots<t_{N}<1$ be such that $\mathbf{t}=\left(t_{1}, \ldots, t_{N}\right) \in B_{\epsilon^{3 / 4}}\left(\mathbf{t}^{0}\right)$. Set

$$
w_{j}(x)=w\left(\frac{x-t_{j}}{\epsilon}\right),
$$

and

$$
r_{0}=\frac{1}{10}\left(\min \left(t_{1}^{0}+1,1-t_{N}^{0}, \frac{1}{2} \min _{i \neq j}\left|t_{i}^{0}-t_{j}^{0}\right|\right)\right) .
$$

Let $\chi: R \rightarrow[0,1]$ be a smooth cut-off function such that $\chi(x)=1$ for $|x|<1$ and $\chi(x)=0$ for $|x|>2$. We now define our approximate solution

$$
\tilde{w}_{j}(x)=w_{j}(x) \chi\left(\frac{x-t_{j}}{r_{0}}\right) .
$$

Then it is easy to see that $\tilde{w}_{j}(x)$ satisfies

$$
\epsilon^{2} \tilde{w}_{j}^{\prime \prime}-\tilde{w}_{j}+\tilde{w}_{j}^{p}=\text { e.s.t. }
$$

in $L^{2}(-1,1)$.

Let $\hat{\xi}(\mathbf{t})=\left(\hat{\xi}_{1}, \ldots, \hat{\xi}_{N}\right)$ be as defined by $(\mathrm{H} 1)$.

Put

$$
w_{\epsilon, \mathbf{t}}(x)=\sum_{j=1}^{N} \hat{\xi}_{j}^{q /(p-1)} \tilde{w}_{j}(x) .
$$


Fix any function $A \in H^{2}(-1,1)$ and define $T[A]$ to be the solution of

$$
\left\{\begin{array}{l}
D T[A]^{\prime \prime}-T[A]+c_{\epsilon} \frac{A^{r}}{(T[A])^{s}}=0, \quad-1<x<1, \\
T[A]^{\prime}(-1)=T[A]^{\prime}(1)=0,
\end{array}\right.
$$

where

$$
c_{\epsilon}=\left(\epsilon \int_{R} w^{r}\right)^{-1}=\xi_{\epsilon}^{\frac{q r}{p-1}-s-1} .
$$

(Recall that $\xi_{\epsilon}$ was first defined in (2.1)). The solution $T[A]$ is unique and positive.

Let $A=w_{\epsilon, \mathbf{t}}$, where $\mathbf{t} \in B_{\epsilon^{3 / 4}}\left(\mathbf{t}^{0}\right)$. Let us now compute

$$
\tau_{i}:=T[A]\left(t_{i}\right) .
$$

From (4.7), we have

$$
\begin{gathered}
\tau_{i}=c_{\epsilon} \int_{-1}^{1} G_{D}\left(t_{i}, z\right) \frac{A^{r}(z)}{(T[A](z))^{s}} d z \\
=c_{\epsilon} \epsilon \sum_{j=1}^{N} \frac{\hat{\xi}_{j}^{p r}}{p-1} \int_{-1}^{1} G_{D}\left(t_{i}, z\right) \tilde{w}_{j}^{r}(z) \tau_{j}^{-s} d z(1+O(\epsilon)) \\
=c_{\epsilon} \epsilon \sum_{j=1}^{N} \hat{\xi}_{j}^{\frac{q r}{p-1}} \tau_{j}^{-s}\left[G_{D}\left(t_{i}, t_{j}\right) \int_{-\infty}^{+\infty} w_{j}^{r}(y) d y+O(\epsilon)\right] \\
\left.=\sum_{j=1}^{N} G_{D}\left(t_{i}, t_{j}\right) \hat{\xi}_{j}^{\frac{q r}{p-1}} \tau_{j}^{-s}+O(\epsilon) \quad \text { (by }(4.8)\right) .
\end{gathered}
$$

Thus we have obtained the following system of equations:

$$
\tau_{i}=\sum_{j=1}^{N} G_{D}\left(t_{i}, t_{j}\right) \hat{\xi}_{j}^{\frac{q r}{p-1}} \tau_{j}^{-s}+O(\epsilon) .
$$

Since the matrix

$$
I+s\left(G_{D}\left(t_{i}, t_{j}\right) \hat{\xi}_{j}^{\frac{q r}{p-1}-s-1}\right)
$$

is clearly nonsingular (note that $G_{D}\left(t_{i}, t_{j}\right)>0$ ), by the implicit function theorem and assumption (H1) the equations (4.10) have a unique solution

$$
\tau_{i}=\hat{\xi}_{i}+O(\epsilon), \quad i=1, \ldots, N .
$$

Hence

$$
T[A]\left(t_{i}\right)=\hat{\xi}_{i}+O(\epsilon) .
$$


Now let $x=t_{i}+\epsilon y$. We calculate for $A=w_{\epsilon, \mathbf{t}}$ :

$$
\begin{aligned}
& T[A](x)-T[A]\left(t_{i}\right)=c_{\epsilon} \int_{-1}^{1}\left[G_{D}(x, z)-G_{D}\left(t_{i}, z\right)\right] \frac{A^{r}}{(T[A])^{s}}(z) d z \\
& =c_{\epsilon} \hat{\xi}_{i}^{\frac{q r}{p-1}} \int_{-1}^{1}\left[G_{D}(x, z)-G_{D}\left(t_{i}, z\right)\right] \frac{\tilde{w}_{i}^{r}}{(T[A])^{s}}(z) d z \\
& +c_{\epsilon} \sum_{j \neq i} \hat{\xi}_{j}^{\frac{q r}{p-1}} \int_{-1}^{1}\left[G_{D}(x, z)-G_{D}\left(t_{i}, z\right)\right] \frac{\tilde{w}_{j}^{r}}{(T[A])^{s}}(z) d z \\
& =c_{\epsilon} \hat{\xi}_{i}^{\frac{q r}{p-1}} \int_{-1}^{1}\left[K_{D}(|x-z|)-K_{D}\left(\left|t_{i}-z\right|\right)\right] \frac{\tilde{w}_{i}^{r}}{(T[A])^{s}}(z) d z \\
& -c_{\epsilon} \hat{\xi}_{i}^{\frac{q r}{p-1}} \int_{-1}^{1}\left[H_{D}(x, z)-H_{D}\left(t_{i}, z\right)\right] \frac{\tilde{w}_{i}^{r}}{(T[A])^{s}}(z) d z \\
& +c_{\epsilon} \sum_{j \neq i} \hat{\xi}_{j}^{\frac{q r}{p-1}} \int_{-1}^{1}\left[G_{D}(x, z)-G_{D}\left(t_{i}, z\right)\right] \frac{\tilde{w}_{j}^{r}}{(T[A])^{s}}(z) d z \quad\left(\text { letting } z=t_{j}+\epsilon y\right) \\
& =\epsilon^{2} c_{\epsilon} \hat{\xi}_{i}^{\frac{q r}{p-1}-s} \int_{-\infty}^{+\infty}\left[\frac{1}{2 D}|z|-\frac{1}{2 D}|y-z|\right] w^{r}(|z|) d z(1+O(\epsilon|y|)) \\
& +\epsilon \hat{\xi}_{i}^{\frac{q r}{p-1}-s}\left[-y \nabla_{t_{i}} H_{D}\left(t_{i}, t_{i}\right)+O\left(\epsilon y^{2}\right)\right] \\
& +\epsilon \sum_{j \neq i}\left[y \nabla_{t_{i}} G_{D}\left(t_{i}, t_{j}\right) \hat{\xi}_{j}^{\frac{q r}{p-1}-s}+O\left(\epsilon y^{2}\right)\right]
\end{aligned}
$$

$$
=\epsilon\left[\hat{\xi}_{i}^{\frac{q r}{p-1}-s} P_{i}(|y|)-\hat{\xi}_{i}^{\frac{q r}{p-1}-s} y \nabla_{t_{i}} H_{D}\left(t_{i}, t_{i}\right)+y \sum_{j \neq i} \nabla_{t_{i}} G_{D}\left(t_{i}, t_{j}\right) \hat{\xi}_{j}^{\frac{q r}{p-1}-s}+O\left(\epsilon y^{2}\right)\right],
$$

where

$$
P_{i}(|y|)=\left(\int_{R} w^{r}\right)^{-1} \int_{-\infty}^{+\infty}\left[\frac{1}{2 D}|z|-\frac{1}{2 D}|y-z|\right] w^{r}(|z|) d z .
$$

Note that $P_{i}$ is an even function.

Let us now define

$$
S[A]:=\epsilon^{2} A^{\prime \prime}-A+\frac{A^{p}}{(T[A])^{q}},
$$


where $T[A]$ is defined by (4.7). Let us choose $A=w_{\epsilon, \mathbf{t}}$ and compute $S\left[w_{\epsilon, \mathbf{t}}\right]$. In fact,

$$
\begin{gathered}
S\left[w_{\epsilon, \mathbf{t}}\right]=\epsilon^{2} w_{\epsilon, \mathbf{t}}^{\prime \prime}-w_{\epsilon, \mathbf{t}}+\frac{w_{\epsilon, \mathbf{t}}^{p}}{\left(T\left[w_{\epsilon, \mathbf{t}}\right]\right)^{q}} \\
=\sum_{j=1}^{N} \hat{\xi}_{j}^{q /(p-1)}\left(\epsilon^{2} \tilde{w}_{j}^{\prime \prime}-\tilde{w}_{j}\right)+\frac{w_{\epsilon, \mathbf{t}}^{p}}{\left(T\left[w_{\epsilon, \mathbf{t}}\right]\right)^{q}}+\text { e.s.t. } \\
=\left[\frac{\left(\sum_{j=1}^{K} \hat{\xi}_{j}^{q /(p-1)} \tilde{w}_{j}\right)^{p}}{\left(T\left[w_{\epsilon, \mathbf{t}}\right]\right)^{q}}-\sum_{j=1}^{K} \hat{\xi}_{j}^{q /(p-1)} \tilde{w}_{j}^{p}\right]+\text { e.s.t. } \\
=E_{1}+E_{2}+\text { e.s.t. }
\end{gathered}
$$

in $L^{2}\left(-\frac{1}{\epsilon}, \frac{1}{\epsilon}\right)$, where

$$
E_{1}=\left[\frac{\left(\sum_{j=1}^{K} \hat{\xi}_{j}^{q /(p-1)} \tilde{w}_{j}\right)^{p}}{\left(T\left[w_{\epsilon, \mathbf{t}}\right]\left(t_{j}\right)\right)^{q}}-\sum_{j=1}^{K} \hat{\xi}_{j}^{q /(p-1)} \tilde{w}_{j}^{p}\right]
$$

and

$$
E_{2}=\left[\frac{\left(\sum_{j=1}^{K} \hat{\xi}_{j}^{q /(p-1)} \tilde{w}_{j}\right)^{p}}{\left(T\left[w_{\epsilon, \mathbf{t}}\right](x)\right)^{q}}-\frac{\left(\sum_{j=1}^{K} \hat{\xi}_{j}^{q /(p-1)} \tilde{w}_{j}\right)^{p}}{\left(T\left[w_{\epsilon, \mathbf{t}}\right]\left(t_{j}\right)^{q}\right.}\right] .
$$

For $E_{1}$ we calculate using (4.11)

$$
\begin{gathered}
E_{1}=\frac{\left(\sum_{j=1}^{N} \hat{\xi}_{j}^{q /(p-1)} \tilde{w}_{j}\right)^{p}}{\left(T\left[w_{\epsilon, \mathbf{t}}\right]\left(t_{j}\right)\right)^{q}}-\sum_{j=1}^{N} \hat{\xi}_{j}^{q /(p-1)} \tilde{w}_{j}^{p} \\
=\sum_{j=1}^{N}\left(\frac{\hat{\xi}_{j}^{q p /(p-1)}}{\hat{\xi}_{j}^{q}+O(\epsilon)}-\hat{\xi}_{j}^{q /(p-1)}\right) \tilde{w}_{j}^{p}=O(\epsilon) \sum_{j=1}^{N} \hat{\xi}_{j}^{q /(p-1)} \tilde{w}_{j}^{p} .
\end{gathered}
$$

Thus we have

$$
\left\|E_{1}\right\|_{L^{2}(-1 / \epsilon, 1 / \epsilon)}=O(\epsilon) .
$$

For $E_{2}$ we calculate

$$
\begin{aligned}
E_{2}=- & \sum_{j=1}^{N} q \frac{\left(\hat{\xi}_{j}^{q /(p-1)} \tilde{w}_{j}\right)^{p}}{\left(T\left[w_{\epsilon, \mathbf{t}}\right]\left(t_{j}\right)\right)^{q+1}}\left(T\left[w_{\epsilon, \mathbf{t}}\right](x)-T\left[w_{\epsilon, \mathbf{t}}\right]\left(t_{j}\right)\right) \\
& +O\left(\sum_{j=1}^{N}\left|T\left[w_{\epsilon, \mathbf{t}}\right]-T\left[w_{\epsilon, \mathbf{t}}\right]\left(t_{j}\right)\right|^{2} \tilde{w}_{j}^{p}\right)
\end{aligned}
$$




$$
\begin{gathered}
=-\sum_{j=1}^{N} q \hat{\xi}_{j}^{q /(p-1)} \tilde{w}_{j}^{p} \frac{T\left[w_{\epsilon, \mathbf{t}}\right]-T\left[w_{\epsilon, \mathbf{t}}\right]\left(t_{j}\right)}{T\left[w_{\epsilon, \mathbf{t}}\right]\left(t_{j}\right)}+O\left(\epsilon^{2} y^{2} \sum_{j=1}^{N} \tilde{w}_{j}^{p}\right) \\
=-\epsilon \sum_{j=1}^{N} q \hat{\xi}_{j}^{\frac{q}{p-1}-1} \tilde{w}_{j}^{p}\left\{\hat{\xi}_{j}^{\frac{q r}{p-1}-s} P_{j}(|y|)\right. \\
\left.-\hat{\xi}_{j}^{\frac{q r}{p-1}-s} y \nabla_{t_{j}} H_{D}\left(t_{j}, t_{j}\right)+y\left[\sum_{l \neq j} \nabla_{t_{j}} G_{D}\left(t_{j}, t_{l}\right) \hat{\xi}_{l}^{\frac{q r}{p-1}-s}\right]\right\}+O\left(\epsilon^{2} y^{2} \sum_{j=1}^{N} \tilde{w}_{j}^{p}\right) .
\end{gathered}
$$

This implies that

$$
\left\|E_{2}\right\|_{L^{2}\left(-\frac{1}{\epsilon}, \frac{1}{\epsilon}\right)}=O(\epsilon) .
$$

Combining (4.19) and (4.21), we conclude that

$$
\left\|S\left[w_{\epsilon, \mathbf{t}}\right]\right\|_{L^{2}\left(-\frac{1}{\epsilon}, \frac{1}{\epsilon}\right)}=O(\epsilon)
$$

The estimates derived in this section provide the main steps that will make our approach work in the rest of the paper.

5. The Liapunov-Schmidt Reduction Method. In this section, we use the Liapunov-Schmidt reduction method to solve the problem

$$
S\left[w_{\epsilon, \mathbf{t}}+v\right]=\sum_{j=1}^{N} \beta_{j} \frac{d \tilde{w}_{j}}{d x}
$$

for real constants $\beta_{j}$ and a function $v \in H^{2}\left(-\frac{1}{\epsilon}, \frac{1}{\epsilon}\right)$ which is small in the corresponding norm, where $\tilde{w}_{i}$ is given by (4.4) and $w_{\epsilon, \mathbf{t}}$ by $(4.6)$.

To this end, we need to study the linearized operator

$$
\tilde{L}_{\epsilon, \mathbf{t}}: H^{2}\left(\Omega_{\epsilon}\right) \rightarrow L^{2}\left(\Omega_{\epsilon}\right)
$$

defined by

$$
\tilde{L}_{\epsilon, \mathbf{t}}:=S_{\epsilon}^{\prime}[A] \phi=\epsilon^{2} \phi^{\prime \prime}-\phi+\frac{p A^{p-1} \phi}{(T[A])^{q}}-q \frac{A^{p}}{(T[A])^{q+1}}\left(T^{\prime}[A] \phi\right),
$$

where $A=w_{\epsilon, \mathbf{t}}, \Omega_{\epsilon}=\left(-\frac{1}{\epsilon}, \frac{1}{\epsilon}\right)$, and for a given $\phi \in L^{2}(\Omega)$ we introduce $T^{\prime}[A] \phi$ as the unique solution of

$$
\left\{\begin{array}{l}
D\left(T^{\prime}[A] \phi\right)^{\prime \prime}-\left(T^{\prime}[A] \phi\right)+c_{\epsilon} r A^{r-1} \phi=0, \quad-1<x<1, \\
\left(T^{\prime}[A] \phi\right)^{\prime}(-1)=\left(T^{\prime}[A] \phi\right)^{\prime}(1)=0 .
\end{array}\right.
$$

We define the approximate kernel and co-kernel, respectively, as follows:

$$
\mathcal{K}_{\epsilon, \mathbf{t}}:=\operatorname{span}\left\{\frac{d \tilde{w}_{i}}{d x} \mid i=1, \ldots, N\right\} \subset H^{2}\left(\Omega_{\epsilon}\right),
$$




$$
\mathcal{C}_{\epsilon, \mathbf{t}}:=\operatorname{span}\left\{\frac{d \tilde{w}_{i}}{d x} \mid i=1, \ldots, N\right\} \subset L^{2}\left(\Omega_{\epsilon}\right),
$$

Recall the definition of the following system of linear operators from (3.5):

$$
\begin{gathered}
L \Phi:=\Delta \Phi-\Phi+p w^{p-1} \Phi \\
-\operatorname{qr}\left(\int_{R} w^{r-1}(I+s \mathcal{B})^{-1} \mathcal{B} \Phi\right)\left(\int_{R} w^{r}\right)^{-1} w^{p},
\end{gathered}
$$

where

$$
\Phi=\left(\begin{array}{l}
\phi_{1} \\
\phi_{2} \\
\vdots \\
\phi_{N}
\end{array}\right) \in\left(H^{2}(R)\right)^{N}
$$

By Lemma 3.3 we know that

$$
L:\left(X_{0} \oplus \cdots \oplus X_{0}\right)^{\perp} \cap\left(H^{2}(R)\right)^{N} \rightarrow\left(X_{0} \oplus \cdots \oplus X_{0}\right)^{\perp} \cap\left(L^{2}(R)\right)^{N}
$$

is invertible with a bounded inverse.

We will see that this system is a limit of the operator $\tilde{L}_{\epsilon, \mathbf{t}}$ as $\epsilon \rightarrow 0$. We also introduce the projection $\pi_{\epsilon, \mathbf{t}}^{\perp}: L^{2}\left(\Omega_{\epsilon}\right) \rightarrow \mathcal{C}_{\epsilon, \mathbf{t}}^{\perp}$ and study the operator $L_{\epsilon, \mathbf{t}}:=\pi_{\epsilon, \mathbf{t}}^{\perp} \circ \tilde{L}_{\epsilon, \mathbf{t}}$. By letting $\epsilon \rightarrow 0$, we will show that $L_{\epsilon, \mathbf{t}}: \mathcal{K}_{\epsilon, \mathbf{t}}^{\perp} \rightarrow \mathcal{C}_{\epsilon, \mathbf{t}}^{\perp}$ is invertible with a bounded inverse provided $\epsilon$ is small enough. This statement is contained in the following proposition.

Proposition 5.1. There exist positive constants $\bar{\epsilon}, \bar{\delta}, \lambda$ such that for all $\epsilon \in(0, \bar{\epsilon})$, $\mathbf{t} \in \Omega^{N}$ with $\min \left(\left|1+t_{1}\right|,\left|1-t_{N}\right|, \min _{i \neq j}\left|t_{i}-t_{j}\right|\right)>\bar{\delta}$,

$$
\left\|L_{\epsilon, \mathbf{t}} \phi\right\|_{L^{2}\left(\Omega_{\epsilon}\right)} \geq \lambda\|\phi\|_{H^{2}\left(\Omega_{\epsilon}\right)} .
$$

Furthermore, the map

$$
L_{\epsilon, \mathbf{t}}=\pi_{\epsilon, \mathbf{t}}^{\perp} \circ \tilde{L}_{\epsilon, \mathbf{t}}: \mathcal{K}_{\epsilon, \mathbf{t}}^{\perp} \rightarrow \mathcal{C}_{\epsilon, \mathbf{t}}^{\perp}
$$

is surjective.

Proof of Theorem 5.1. This proof follows the method of Liapunov-Schmidt reduction which was also used in [3], [4], [12], [13], [10], [23], [24], [33] and [36].

Suppose (5.4) is false. Then there exist sequences $\left\{\epsilon_{k}\right\},\left\{\mathbf{t}^{k}\right\},\left\{\phi^{k}\right\}$ with $\epsilon_{k} \rightarrow 0, \mathbf{t}^{k} \in$ $\Omega^{N}, \min \left(\left|1+t_{1}^{k}\right|,\left|1-t_{N}^{k}\right|, \min _{i \neq j}\left|t_{i}^{k}-t_{j}^{k}\right|\right)>\bar{\delta}, \phi^{k}=\phi_{\epsilon_{k}} \in K_{\epsilon_{k}, \mathbf{t}^{k}}^{\perp}, k=1,2, \ldots$ such that

$$
\begin{aligned}
& \left\|L_{\epsilon_{k}, \mathbf{t}^{k}} \phi^{k}\right\|_{L^{2}\left(\Omega_{\epsilon_{k}}\right)} \rightarrow 0, \quad \text { as } k \rightarrow \infty, \\
& \left\|\phi^{k}\right\|_{H^{2}\left(\Omega_{\epsilon_{k}}\right)}=1, \quad k=1,2, \ldots
\end{aligned}
$$

We define $\phi_{\epsilon, i}, i=1,2, \ldots, N$ and $\phi_{\epsilon, N+1}$ as follows:

$$
\begin{gathered}
\phi_{\epsilon, i}(x)=\phi_{\epsilon}(x) \chi\left(\frac{x-t_{i}}{r_{0}}\right), \quad x \in \Omega, \\
\phi_{\epsilon, N+1}(x)=\phi_{\epsilon}(x)-\sum_{i=1}^{N} \phi_{\epsilon, i}(x), \quad x \in \Omega .
\end{gathered}
$$


At first (after rescaling) $\phi_{\epsilon, i}$ are only defined on $\Omega_{\epsilon}$. However, by a standard result they can be extended to $R$ such that their norm in $H^{2}(R)$ is still bounded by a constant independent of $\epsilon$ and $\mathbf{t}$ for $\epsilon$ small enough. In the following we will study this extension. For simplicity of notation we keep the same notation for the extension. Since for $i=1,2, \ldots, N$ each sequence $\left\{\phi_{i}^{k}\right\}:=\left\{\phi_{\epsilon_{k}, i}\right\}(k=1,2, \ldots)$ is bounded in $H_{l o c}^{2}(R)$ it has a weak limit in $H_{l o c}^{2}(R)$, and therefore also a strong limit in $L_{l o c}^{2}(R)$ and $L_{l o c}^{\infty}(R)$. Call these limits $\phi_{i}$. Then $\phi=\left(\begin{array}{c}\phi_{1} \\ \phi_{2} \\ \vdots \\ \phi_{N}\end{array}\right)$ solves the system

$$
L \phi=0 .
$$

By Lemma 3.3, $\phi \in \operatorname{Ker}(L)=X_{0} \oplus \cdots \oplus X_{0}$. Since $\phi^{k} \in K_{\epsilon_{k}, x_{k}}^{\perp}$ by taking $k \rightarrow \infty$ we get $\phi \in \operatorname{Ker}(L)^{\perp}$. Therefore, $\phi=0$.

By elliptic estimates we get $\left\|\phi_{\epsilon_{k}, i}\right\|_{H^{2}(R)} \rightarrow 0$ as $k \rightarrow \infty$ for $i=1,2, \ldots, N$.

Furthermore, $\phi_{\epsilon, N+1} \rightarrow \phi_{N+1}$ in $H^{2}(R)$, where $\Phi_{N+1}$ satisfies

$$
\Delta \phi_{N+1}-\phi_{N+1}=0 \quad \text { in } R \text {. }
$$

Therefore we conclude $\phi_{N+1}=0$ and $\left\|\phi_{N+1}^{k}\right\|_{H^{2}(R)} \rightarrow 0$ as $k \rightarrow \infty$.

This contradicts $\left\|\phi^{k}\right\|_{H^{2}\left(\Omega_{\epsilon_{k}}\right)}=1$. To complete the proof of Proposition 5.1 we just need to show that the operator which is conjugate to $L_{\epsilon, \mathbf{t}}$ (denoted by $L_{\epsilon, \mathbf{t}}^{*}$ ) is injective from $\mathcal{K}_{\epsilon, \mathbf{t}}^{\perp}$ to $\mathcal{C}_{\epsilon, \mathbf{t}}^{\perp}$. Note that $L_{\epsilon, \mathbf{t}}^{*} \psi=\pi_{\epsilon, \mathbf{t}} \circ \tilde{L}_{\epsilon, \mathbf{t}}^{*}$ with

$$
\tilde{L}_{\epsilon, \mathbf{t}}^{*} \psi=\epsilon^{2} \Delta \psi-\psi+\frac{p A^{p-1} \psi}{(T[A])^{q}}-q T^{\prime}[A]\left(\frac{A^{p} \psi}{(T[A])^{q+1}}\right) .
$$

The proof for $L_{\epsilon, \mathbf{t}}^{*}$ follows exactly along the same lines as the proof for $L_{\epsilon, \mathbf{t}}$ and is therefore omitted.

Now we are in a position to solve the equation

$$
\pi_{\epsilon, \mathbf{t}}^{\perp} \circ S_{\epsilon}\left(w_{\epsilon, \mathbf{t}}+\phi\right)=0 .
$$

Since $\left.L_{\epsilon, \mathbf{t}}\right|_{K_{\epsilon, \mathbf{t}}^{\perp}}$ is invertible (call the inverse $L_{\epsilon, \mathbf{t}}^{-1}$ ) we can rewrite this as

$$
\phi=-\left(L_{\epsilon, \mathbf{t}}^{-1} \circ \pi_{\epsilon, \mathbf{t}}^{\perp} \circ S_{\epsilon}\left(w_{\epsilon, \mathbf{t}}\right)\right)-\left(L_{\epsilon, \mathbf{t}}^{-1} \circ \pi_{\epsilon, \mathbf{t}}^{\perp} \circ N_{\epsilon, \mathbf{t}}(\phi)\right) \equiv M_{\epsilon, \mathbf{t}}(\phi),
$$

where

$$
N_{\epsilon, \mathbf{t}}(\phi)=S_{\epsilon}\left(w_{\epsilon, \mathbf{t}}+\phi\right)-S_{\epsilon}\left(w_{\epsilon, \mathbf{t}}\right)-S_{\epsilon}^{\prime}\left(w_{\epsilon, \mathbf{t}}\right) \phi
$$

and the operator $M_{\epsilon, \mathbf{t}}$ is defined by (5.9) for $\phi \in H^{2}\left(\Omega_{\epsilon}\right)$. We are going to show that the operator $M_{\epsilon, \mathbf{t}}$ is a contraction on

$$
B_{\epsilon, \delta} \equiv\left\{\phi \in H^{2}\left(\Omega_{\epsilon}\right) \mid\|\phi\|_{H^{2}\left(\Omega_{\epsilon}\right)}<\delta\right\}
$$

if $\delta$ and $\epsilon$ are small enough. We have by (4.22) and Proposition 5.1

$$
\begin{gathered}
\left\|M_{\epsilon, \mathbf{t}}(\phi)\right\|_{H^{2}\left(\Omega_{\epsilon}\right)} \leq \lambda^{-1}\left(\left\|\pi_{\epsilon, \mathbf{t}}^{\perp} \circ N_{\epsilon, \mathbf{t}}(\phi)\right\|_{L^{2}\left(\Omega_{\epsilon}\right)}\right. \\
\left.+\left\|\pi_{\epsilon, \mathbf{t}}^{\perp} \circ S_{\epsilon}\left(w_{\epsilon, \mathbf{t}}\right)\right\|_{L^{2}\left(\Omega_{\epsilon}\right)}\right)
\end{gathered}
$$




$$
\leq \lambda^{-1} C(c(\delta) \delta+\epsilon)
$$

where $\lambda>0$ is independent of $\delta>0, \epsilon>0$ and $c(\delta) \rightarrow 0$ as $\delta \rightarrow 0$. Similarly we show

$$
\begin{gathered}
\left\|M_{\epsilon, \mathbf{t}}(\phi)-M_{\epsilon, \mathbf{t}}\left(\phi^{\prime}\right)\right\|_{H^{2}\left(\Omega_{\epsilon}\right)} \\
\leq \lambda^{-1} C(c(\delta) \delta)\left\|\phi-\phi^{\prime}\right\|_{H^{2}\left(\Omega_{\epsilon}\right)},
\end{gathered}
$$

where $c(\delta) \rightarrow 0$ as $\delta \rightarrow 0$. If we choose $\delta=\epsilon^{\alpha}$ for $\alpha<1$ and $\epsilon$ small enough, then $M_{\epsilon, \mathbf{t}}$ is a contraction on $B_{\epsilon, \delta}$. The existence of a fixed point $\phi_{\epsilon, \mathbf{t}}$ now follows from the standard contraction mapping principle and $\phi_{\epsilon, \mathbf{t}}$ is a solution of (5.9).

We have thus proved

LEMma 5.2. There exist $\bar{\epsilon}>0 \bar{\delta}>0$ such that for every pair of $\epsilon, \mathbf{t}$ with $0<\epsilon<\bar{\epsilon}$ and $\mathbf{t} \in \Omega^{N}, 1+t_{1}>\bar{\delta}, 1-t_{N}>\bar{\delta}, \frac{1}{2}\left|t_{i}-t_{j}\right|>\bar{\delta}$ there is a unique $\phi_{\epsilon, \mathbf{t}} \in K_{\epsilon, \mathbf{t}}^{\perp}$ satisfying $S_{\epsilon}\left(w_{\epsilon, \mathbf{t}}+\phi_{\epsilon, \mathbf{t}}\right) \in \mathcal{C}_{\epsilon, \mathbf{t}}$. Furthermore, we have the estimate

$$
\left\|\phi_{\epsilon, \mathbf{t}}\right\|_{H^{2}\left(\Omega_{\epsilon}\right)} \leq C \epsilon^{\alpha}
$$

where $\alpha<1$.

REMARK 5.1. By one more iteration, it can actually be shown that

$$
\left\|\phi_{\epsilon, \mathbf{t}}\right\|_{H^{2}\left(\Omega_{\epsilon}\right)} \leq C \epsilon .
$$

6. The reduced problem. In this section we solve the reduced problem and prove our main existence result given by Theorem 2.1. that

By Lemma 5.2, for every $\mathbf{t} \in B_{\epsilon^{3 / 4}}\left(\mathbf{t}_{0}\right)$, there exists a unique solution $\phi_{\epsilon, \mathbf{t}} \in \mathcal{K}_{\epsilon, \mathbf{t}}^{\perp}$ such

$$
S\left[w_{\epsilon, \mathbf{t}}+\phi_{\epsilon, \mathbf{t}}\right]=v_{\epsilon, \mathbf{t}} \in \mathcal{C}_{\epsilon, \mathbf{t}}
$$

Our idea is to find $\mathbf{t}^{\epsilon}=\left(t_{1}^{\epsilon}, \ldots, t_{N}^{\epsilon}\right)$ near $\mathbf{t}^{0}$ such that also

$$
S\left[w_{\epsilon, \mathbf{t}^{\epsilon}}+\phi_{\epsilon, \mathbf{t}^{\epsilon}}\right] \perp \mathcal{C}_{\epsilon, \mathbf{t}^{\epsilon}}
$$

(and therefore $S\left[w_{\epsilon, \mathbf{t}^{\epsilon}}+\phi_{\epsilon, \mathbf{t}^{\epsilon}}\right]=0$ ).

To this end, we let

$$
\begin{aligned}
& W_{\epsilon, i}(\mathbf{t}):=\epsilon^{-1}\left(\hat{\xi}_{i}\right)^{1-\frac{q}{p-1}} \int_{-1}^{1} S\left[w_{\epsilon, \mathbf{t}}+\phi_{\epsilon, \mathbf{t}}\right] \frac{d \tilde{w}_{i}}{d x} d x, \\
& W_{\epsilon}(\mathbf{t}):=\left(W_{\epsilon, 1}(\mathbf{t}), \ldots, W_{\epsilon, N}(\mathbf{t})\right): B_{\epsilon^{3 / 4}}\left(\mathbf{t}_{0}\right) \rightarrow R^{N} .
\end{aligned}
$$

Then $W_{\epsilon}(\mathbf{t})$ is a map which is continuous in $\mathbf{t}$ and our problem is reduced to finding a zero of the vector field $W_{\epsilon}(\mathbf{t})$.

Let us now calculate $W_{\epsilon}(\mathbf{t})$.

We calculate:

$$
W_{\epsilon, i}(\mathbf{t})=\epsilon^{-1}\left(\hat{\xi}_{i}\right)^{1-\frac{q}{p-1}} \int_{-1}^{1} S\left[w_{\epsilon, \mathbf{t}}+\phi_{\epsilon, \mathbf{t}}\right] \frac{d \tilde{w}_{i}}{d x}
$$




$$
\begin{gathered}
=\epsilon^{-1}\left(\hat{\xi}_{i}\right)^{1-\frac{q}{p-1}} \int_{-1}^{1} S\left[w_{\epsilon, \mathbf{t}}\right] \frac{d \tilde{w}_{i}}{d x} \\
+\epsilon^{-1}\left(\hat{\xi}_{i}\right)^{1-\frac{q}{p-1}} \int_{-1}^{1} S_{\epsilon}^{\prime}\left[w_{\epsilon, \mathbf{t}}\right] \phi_{\epsilon, \mathbf{t}} \frac{d \tilde{w}_{i}}{d x} \\
+\epsilon^{-1}\left(\hat{\xi}_{i}\right)^{1-\frac{q}{p-1}} \int_{-1}^{1} N_{\epsilon}\left(\phi_{\epsilon, \mathbf{t}}\right) \frac{d \tilde{w}_{i}}{d x} \\
=I_{1}+I_{2}+I_{3},
\end{gathered}
$$

where $I_{1}, I_{2}$ and $I_{3}$ are defined by the last equality.

The computation of $I_{3}$ is the easiest: note that by Taylor expansion for (5.10), the first term in the expansion of $N_{\epsilon}$ is quadratic in $\phi_{\epsilon, \mathbf{t}}$. So

$$
I_{3}=O(\epsilon) \text {. }
$$

We will now compute $I_{1}$ and $I_{2}$. The result will be that $I_{1}$ is the leading term and $I_{2}=O(\epsilon)$.

For $I_{1}$, we have

$$
I_{1}=\epsilon^{-1}\left(\hat{\xi}_{i}\right)^{1-\frac{q}{p-1}} \int_{-1}^{1}\left(E_{1}+E_{2}\right) \frac{d \tilde{w}_{i}}{d x} d x=\epsilon^{-1}\left(\hat{\xi}_{i}\right)^{1-\frac{q}{p-1}} \int_{-1}^{1} E_{2} \frac{d \tilde{w}_{i}}{d x} d x+O(\epsilon),
$$

where $E_{1}$ and $E_{2}$ were defined in (4.16) and (4.17), respectively, using that $E_{1}$ is an even function.

We calculate by $(4.20)$

$$
\begin{aligned}
& \epsilon^{-1}\left(\hat{\xi}_{i}\right)^{1-\frac{q}{p-1}} \int_{-1}^{1} E_{2} \frac{d \tilde{w}_{i}}{d x} d x \\
& =-q\left[\sum_{j=1}^{N} \nabla_{t_{i}} G_{D}\left(t_{i}, t_{j}\right) \hat{\xi}_{j}^{\frac{q r}{p-1}-s}\right] \int_{R} y w^{p}(y) w^{\prime}(y) d y+O(\epsilon) \\
& =\left(\frac{q}{p+1} \int_{R} w^{p+1}\right)\left[\sum_{j=1}^{N} \nabla_{t_{i}} G_{D}\left(t_{i}, t_{j}\right) \hat{\xi}_{j}^{\frac{q r}{p-1}-s}\right]+O(\epsilon) .
\end{aligned}
$$

Thus we have

$$
I_{1}=\frac{q}{p+1} \int_{R} w^{p+1}(y) d y\left[\sum_{j=1}^{N} \nabla_{t_{i}} G_{D}\left(t_{i}, t_{j}\right) \hat{\xi}_{j}^{\frac{q r}{p-1}-s}\right]+O(\epsilon) .
$$

For $I_{2}$ we calculate

$$
\epsilon\left(\hat{\xi}_{i}\right)^{\frac{q}{p-1}-1} I_{2}=\int_{-1}^{1} S^{\prime}\left[w_{\epsilon, \mathbf{t}}\right]\left(\phi_{\epsilon, \mathbf{t}}\right) \frac{d \tilde{w}_{i}}{d x}
$$




$$
\begin{gathered}
\int_{-1}^{1}\left[\epsilon^{2} \Delta \phi_{\epsilon, \mathbf{t}}-\phi_{\epsilon, \mathbf{t}}+\frac{p w_{\epsilon, \mathbf{t}}^{p-1} \phi_{\epsilon, \mathbf{t}}}{\left(T\left[w_{\epsilon, \mathbf{t}}\right]\right)^{q}}-q \frac{w_{\epsilon, \mathbf{t}}^{p}}{\left(T\left[w_{\epsilon, \mathbf{t}}\right]\right)^{q+1}}\left(T^{\prime}\left[w_{\epsilon, \mathbf{t}}\right] \phi_{\epsilon, \mathbf{t}}\right)\right] \frac{d \tilde{w}_{i}}{d x} \\
=\int_{-1}^{1}\left[\epsilon^{2} \Delta \frac{d \tilde{w}_{i}}{d x}-\frac{d \tilde{w}_{i}}{d x}+\frac{d \tilde{w}_{i}}{d x} \frac{p w_{\epsilon, \mathbf{t}}^{p-1}}{\left(T\left[w_{\epsilon, \mathbf{t}}\right]\right)^{q}}\right] \phi_{\epsilon, \mathbf{t}} \\
-q \int_{-1}^{1} \frac{w_{\epsilon, \mathbf{t}}^{p}}{\left(T\left[w_{\epsilon, \mathbf{t}}\right]\right)^{q+1}}\left(T^{\prime}\left[w_{\epsilon, \mathbf{t}}\right] \phi_{\epsilon, \mathbf{t}}\right) \frac{d \tilde{w}_{i}}{d x} \\
=\int_{-1}^{1}\left(p \frac{\hat{\xi}_{i}^{q} \tilde{w}_{i}^{p-1}}{\left(T\left[w_{\epsilon, \mathbf{t}}\right]\right)^{q}}-p \tilde{w}_{i}^{p-1}\right) \phi_{\epsilon, \mathbf{t}} \frac{d \tilde{w}_{i}}{d x} \\
-q \int_{-1}^{1} \frac{w_{\epsilon, \mathbf{t}}^{p}}{\left(T\left[w_{\epsilon, \mathbf{t}}\right]\right)^{q+1}}\left(T^{\prime}\left[w_{\epsilon, \mathbf{t}}\right] \phi_{\epsilon, \mathbf{t}}\right) \frac{d \tilde{w}_{i}}{d x}=O\left(\epsilon^{2}\right)
\end{gathered}
$$

since

$$
\begin{gathered}
\left\|\left(\frac{p \hat{\xi}_{i}^{q} \tilde{w}_{i}^{p-1}}{\left(T\left[w_{\epsilon, \mathbf{t}}\right]\right)^{q}}-p \tilde{w}_{i}^{p-1}\right) \phi_{\epsilon, \mathbf{t}}\right\|_{L^{2}\left(\Omega_{\epsilon}\right)}=O(\epsilon), \\
\left\|\phi_{\epsilon, \mathbf{t}}\right\|_{H^{2}\left(\Omega_{\epsilon}\right)}=O(\epsilon), \\
T^{\prime}\left[w_{\epsilon, \mathbf{t}}\right]\left(\phi_{\epsilon, \mathbf{t}}\right)\left(t_{i}\right)=O(\epsilon), \\
T^{\prime}\left[w_{\epsilon, \mathbf{t}}\right]\left(\phi_{\epsilon, \mathbf{t}}\right)\left(t_{i}+\epsilon y\right)-T^{\prime}\left[w_{\epsilon, \mathbf{t}}\right]\left(\phi_{\epsilon, \mathbf{t}}\right)\left(t_{i}\right)=O\left(\epsilon^{2}|y|\right) .
\end{gathered}
$$

Combining $I_{1}$ and $I_{2}$, we have

$$
\begin{gathered}
W_{\epsilon, i}(\mathbf{t})=\frac{q}{p+1} \int_{R} w^{p+1} \times\left[\sum_{j=1}^{N} \nabla_{t_{i}} G_{D}\left(t_{i}, t_{j}\right) \hat{\xi}_{j}^{\frac{q r}{p-1}-s}\right]+O(\epsilon) \\
=\frac{q}{p+1} \int_{R} w^{p+1} F_{i}(\mathbf{t})+O(\epsilon),
\end{gathered}
$$

where $F_{i}(\mathbf{t})$ was defined in (2.10).

By our assumption (H3), at $\mathbf{t}^{0}$, we have $F\left(\mathbf{t}^{0}\right)=0$ and

$$
\operatorname{det}\left(\nabla_{\mathbf{t}^{0}} F\left(\mathbf{t}^{0}\right)\right) \neq 0 .
$$
by

$$
c_{1}=-\frac{q}{p+1} \int_{R} w^{p+1} .
$$


Then Brouwer's fixed point theorem shows that for $\epsilon<<1$ there exists a $\mathbf{t}^{\epsilon}$ such that $W_{\epsilon}\left(\mathbf{t}^{\epsilon}\right)=0$ and $\mathbf{t}^{\epsilon} \in B_{\epsilon^{3 / 4}}\left(\mathbf{t}^{0}\right)$.

Thus we have proved the following proposition.

Proposition 6.1. For $\epsilon$ sufficiently small there exist points $\mathbf{t}^{\epsilon}$ with $\mathbf{t}^{\epsilon} \rightarrow \mathbf{t}^{0}$ such that $W_{\epsilon}\left(\mathbf{t}^{\epsilon}\right)=0$.

REMARK 6.1. A more detailed computation reveals that

$$
\left|\mathbf{t}^{\epsilon}-\mathbf{t}^{0}\right|=O(\epsilon) .
$$

Finally, we prove Theorem 2.1.

Proof of Theorem 2.1. By Proposition 6.1, there exists $\mathbf{t}^{\epsilon} \rightarrow \mathbf{t}^{0}$ such that $W_{\epsilon}\left(\mathbf{t}^{\epsilon}\right)=0$. In other words, $S\left[w_{\epsilon, \mathbf{t}^{\epsilon}}+\phi_{\epsilon, \mathbf{t}^{\epsilon}}\right]=0$. Let $A_{\epsilon}=\xi_{\epsilon}^{\frac{q}{p-1}}\left(w_{\epsilon, \mathbf{t}^{\epsilon}}+\phi_{\epsilon, \mathbf{t}^{\epsilon}}\right), H_{\epsilon}=\xi_{\epsilon} T\left[w_{\epsilon, \mathbf{t}^{\epsilon}}+\phi_{\epsilon, \mathbf{t}^{\epsilon}}\right]$. By the Maximum Principle, $A_{\epsilon}>0, H_{\epsilon}>0$. Moreover $\left(A_{\epsilon}, H_{\epsilon}\right)$ satisfies all the properties of Theorem 2.1.

7. Classifying the $N$-peaked solutions: proof of Theorem 2.2. Let $\left(A_{\epsilon}, H_{\epsilon}\right)$ be a solution of (1.7) satisfying (2.33) and (2.34). We now show that $\left(A_{\epsilon}, H_{\epsilon}\right)$ is generated exactly by two types of peaks, that is, we prove Theorem 2.2. First we make the following scaling

$$
A_{\epsilon}=\xi_{\epsilon}^{\frac{q}{p-1}} \hat{A}_{\epsilon}, H_{\epsilon}=\xi_{\epsilon} \hat{H}_{\epsilon}
$$

where $\xi_{\epsilon}$ is defined at $(2.1)$. Hence $\left(\hat{A}_{\epsilon}, \hat{H}_{\epsilon}\right)$ satisfies

$$
\left\{\begin{array}{l}
\epsilon^{2} \Delta \hat{A}_{\epsilon}-\hat{A}_{\epsilon}+\frac{\hat{A}_{\epsilon}^{p}}{\hat{H}_{\epsilon}^{q}}=0,-1<x<1, \\
D \Delta \hat{H}_{\epsilon}-\hat{H}_{\epsilon}+c_{\epsilon} \frac{\hat{A}_{\epsilon}^{r}}{\hat{H}_{\epsilon}^{s}}=0,-1<x<1,
\end{array}\right.
$$

where $c_{\epsilon}$ is defined in (4.8).

Now (2.33) and (2.34) imply that

$$
\hat{A}_{\epsilon} \sim \sum_{j=1}^{N}\left(\hat{\xi}_{j}^{\epsilon}\right)^{\frac{q}{p-1}} w\left(\frac{x-t_{j}^{\epsilon}}{\epsilon}\right), \quad \hat{H}_{\epsilon}\left(t_{j}^{\epsilon}\right)=\hat{\xi}_{j}^{\epsilon} .
$$

Letting $\epsilon \rightarrow 0$, we assume that

$$
\hat{\xi}_{j}^{\epsilon} \rightarrow \hat{\xi}_{j}^{0}, \quad t_{j}^{\epsilon} \rightarrow t_{j}^{0}, \quad j=1, \ldots, N .
$$

We see that $\hat{H}_{\epsilon} \rightarrow h_{0}(x)$ where $h_{0}(x)$ satisfies

$$
\left\{\begin{array}{l}
D \Delta h_{0}-h_{0}+\sum_{j=1}^{N}\left(\hat{\xi}_{j}^{0}\right)^{\frac{q r}{p-1}-s} \delta\left(x-t_{j}^{0}\right)=0,-1<x<1, \\
h_{0}^{\prime}(-1)=h_{0}^{\prime}(1)=0 .
\end{array}\right.
$$

In other words, we have

$$
h_{0}(x)=\sum_{j=1}^{N}\left(\hat{\xi}_{j}^{0}\right)^{\frac{q r}{p-1}-s} G_{D}\left(x, t_{j}^{0}\right) .
$$


Since $h_{0}\left(t_{j}^{0}\right)=\hat{\xi}_{j}^{0}, j=1, \ldots, N$, we have from $(7.4)$ that $\left(\hat{\xi}_{1}^{0}, \ldots, \hat{\xi}_{N}^{0}\right)$ must satisfy the following identity:

$$
\sum_{j=1}^{N} G_{D}\left(t_{i}^{0}, t_{j}^{0}\right)\left(\hat{\xi}_{j}^{0}\right)^{\frac{q r}{p-1}-s}=\hat{\xi}_{i}^{0}, \quad i=1, \ldots, N
$$

This is the same as (2.5).

Define

$$
\tilde{A}_{\epsilon, j}=\hat{A}_{\epsilon} \chi\left(\frac{x-t_{j}^{0}}{\tilde{r}_{0}}\right)
$$

where $\tilde{r}_{0}$ is a very small number. Then $\tilde{A}_{\epsilon, j}$ is supported in the interval $I_{j}^{\epsilon}=\left(-\tilde{r}_{0}+t_{j}^{\epsilon}, \tilde{r}_{0}+t_{j}^{\epsilon}\right)$. We may choose $\tilde{r}_{0}$ so small that $I_{i}^{\epsilon} \cap I_{j}^{\epsilon}=\emptyset$ for $i \neq j$. Then

$$
\hat{A}_{\epsilon}=\sum_{j=1}^{N} \tilde{A}_{\epsilon, j}+\text { e.s.t. }
$$

Now we multiply the first equation in $(7.1)$ by $\tilde{A}_{\epsilon, j}^{\prime}$ and integrate over $(-1,1)$. We obtain

$$
\begin{gathered}
0=\int_{-1}^{1}\left[\left(\frac{\hat{A}_{\epsilon}{ }^{p}}{\hat{H}_{\epsilon}^{q}}\right) \tilde{A}_{\epsilon, j}^{\prime}-\left(\frac{\hat{A}_{\epsilon}^{p}}{\hat{H}_{\epsilon}^{q}}\right)^{\prime} \tilde{A}_{\epsilon, j}\right] \\
=-2 \int_{I_{j}^{\epsilon}}\left(\frac{\hat{A}_{\epsilon}^{p}}{\hat{H}_{\epsilon}^{q}}\right)^{\prime} \hat{A}_{\epsilon}+\text { e.s.t. } \\
=-2 \int_{I_{j}^{\epsilon}}\left[\frac{p \hat{A}_{\epsilon}^{p} \hat{A}_{\epsilon}^{\prime}}{\hat{H}_{\epsilon}^{q}}-\frac{q \hat{A}_{\epsilon}^{p+1} \hat{H}_{\epsilon}^{\prime}}{\hat{H}_{\epsilon}^{q+1}}\right]+\text { e.s.t. } \\
=\frac{q(p+2)}{p+1} \int_{I_{j}^{\epsilon}} \frac{\hat{A}_{\epsilon}^{p+1}}{\hat{H}_{\epsilon}^{q+1}} \hat{H}_{\epsilon}^{\prime}+\text { e.s.t. }
\end{gathered}
$$

By the equation for $\hat{H}_{\epsilon}$, we have that

$$
\hat{H}_{\epsilon}(x)=c_{\epsilon} \int_{-1}^{1} G_{D}(x, z) \frac{\hat{A}_{\epsilon}^{r}}{\hat{H}_{\epsilon}^{s}}
$$

and thus for $x \in I_{j}^{\epsilon}$,

$$
\hat{H}_{\epsilon}(x)=\sum_{k=1}^{N} G_{D}\left(x, t_{k}^{\epsilon}\right)\left(\hat{\xi}_{k}^{\epsilon}\right)^{\frac{q r}{p-1}-s}+O(\epsilon)
$$

and

$$
\hat{H}_{\epsilon}^{\prime}\left(t_{j}^{\epsilon}\right)=\sum_{k=1}^{N} \nabla_{t_{j}^{\epsilon}} G_{D}\left(t_{j}^{\epsilon}, t_{k}^{\epsilon}\right)\left(\hat{\xi}_{k}^{\epsilon}\right)^{\frac{q r}{p-1}-s}+O(\epsilon) .
$$


Substituting (7.7) into (7.6) and using (7.2), we obtain the following identity

$$
\sum_{k=1}^{N} \nabla_{t_{j}^{\epsilon}} G_{D}\left(t_{j}^{\epsilon}, t_{k}^{\epsilon}\right)\left(\hat{\xi}_{k}^{\epsilon}\right)^{\frac{q r}{p-1}-s}=o(1)
$$

and hence

$$
\sum_{k=1}^{N} \nabla_{t_{j}^{0}} G_{D}\left(t_{j}^{0}, t_{k}^{0}\right)\left(\hat{\xi}_{k}^{0}\right)^{\frac{q r}{p-1}-s}=0, j=1, \ldots, N
$$

which is the same as (2.12).

Note that by the expression for $h_{0}$ in (7.4), (7.9) is equivalent to the following

$$
h_{0}^{\prime}\left(t_{j}^{0}+\right)+h_{0}^{\prime}\left(t_{j}^{0}-\right)=0, j=1, \ldots, N,
$$

where $h_{0}^{\prime}\left(t_{j}^{0}+\right)$ is the right-hand derivative of $h_{0}$ at $t_{j}^{0}$ and $h_{0}^{\prime}\left(t_{j}^{0}-\right)$ is the left-hand derivative of $h_{0}$ at $t_{j}^{0}$. On the other hand, from the equation for $h_{0}$, we have that

$$
D\left(h_{0}^{\prime}\left(t_{j}^{0}+\right)-h_{0}^{\prime}\left(t_{j}^{0}-\right)\right)=-\left(\hat{\xi}_{j}^{0}\right)^{\frac{q r}{p-1}-s}, j=1, \ldots, N .
$$

Solving (7.10) and (7.11), we have that

$$
h_{0}^{\prime}\left(t_{j}^{0}+\right)=-h_{0}^{\prime}\left(t_{j}^{0}-\right)=-\frac{1}{2 D}\left(\hat{\xi}_{j}^{0}\right)^{\frac{q r}{p-1}-s}<0, j=1, \ldots, N .
$$

Since $h_{0}$ satisfies $D h_{0}^{\prime \prime}=h_{0}>0$ in each interval $\left(t_{j-1}^{0}, t_{j}^{0}\right), j=2, \ldots, N$, we see that there exists a unique point $s_{j-1} \in\left(t_{j-1}^{0}, t_{j}^{0}\right)$ such that $h_{0}^{\prime}\left(s_{j-1}\right)=0$. Since $h_{0}^{\prime}(-1)=0$, by using symmetry, we see that

$$
\frac{s_{j-1}+s_{j}}{2}=t_{j}^{0}, j=1, \ldots, N,
$$

where we take $s_{0}=-1, s_{N}=1$. Let $2 l_{j}=s_{j}-s_{j-1}, j=1, \ldots, N$. Note that on each interval $\left(-l_{j}+t_{j}^{0}, l_{j}+t_{j}^{0}\right), h_{0}$ satisfies $D \Delta h_{0}-h_{0}+\left(\hat{\xi}_{j}^{0}\right)^{\frac{q r}{p-1}-s} \delta\left(t-t_{j}^{0}\right)=0$ with Neumann boundary conditions at both ends. Thus from (1.4) it is easy to see that

$$
\begin{gathered}
\left(\hat{\xi}_{j}^{0}\right)^{\frac{q r}{p-1}-s-1}=2 \sqrt{D} \tanh \left(\frac{l_{j}}{\sqrt{D}}\right), j=1, \ldots, N, \\
h_{0}\left(l_{j}\right)=\frac{\hat{\xi}_{j}^{0}}{\cosh \left(\frac{l_{j}}{\sqrt{D}}\right)} .
\end{gathered}
$$

Since $h_{0}$ is continuous on $(-1,1)$, we have

$$
h_{0}\left(l_{1}\right)=h_{0}\left(l_{2}\right)=\ldots=h_{0}\left(l_{N}\right) .
$$

Using (7.14) and (7.15), we see that (7.16) is equivalent to

$$
b\left(\frac{l_{1}}{\sqrt{D}}\right)=b\left(\frac{l_{2}}{\sqrt{D}}\right)=\ldots=b\left(\frac{l_{N}}{\sqrt{D}}\right),
$$

where the function $b$ was defined in (2.25). Suppose without loss of generality that $l_{1} \leq l_{2}$, then we take $l_{1}=l$ and (7.17) implies that $l_{2} \in\{l, \bar{l}\}$ and that $l_{j} \in\{l, \bar{l}\}$ for $j=2, \ldots, N$. Thus $l$ must satisfy (2.26) and (2.27).

This finishes the proof of Theorem 2.2.

REMARK 7.1. The proof of Theorem 2.2 implies that if $\mathbf{t}^{0}=\left(t_{1}^{0}, \ldots, t_{N}^{0}\right)$ satisfies (H1) and (2.12), then necessarily, we have $t_{j}^{0}-t_{j-1}^{0}=l_{j} \in\{l, \bar{l}\}$. That is, there are at most $2^{N}$ solutions satisfying (H1)-(H3). 
8. Stability Analysis: Large Eigenvalues. In this section, we study the eigenvalues with $\lambda_{\epsilon} \rightarrow \lambda_{0} \neq 0$ as $\epsilon \rightarrow 0$ (or, more precisely, with nonzero accumulation points).

We need to analyze the following eigenvalue problem

$$
\begin{aligned}
\tilde{L}_{\epsilon, \mathbf{t}^{\epsilon}} \phi_{\epsilon}=\epsilon^{2} \phi_{\epsilon}^{\prime \prime}-\phi_{\epsilon} & +\frac{p A_{\epsilon}^{p-1} \phi_{\epsilon}}{\left(T\left[A_{\epsilon}\right]\right)^{q}}-q \frac{A_{\epsilon}^{p}}{\left(T\left[A_{\epsilon}\right]\right)^{q+1}} \psi_{\epsilon} \\
& =\lambda_{\epsilon} \phi_{\epsilon},
\end{aligned}
$$

where $\psi_{\epsilon}$ satisfies

$$
D \psi_{\epsilon}^{\prime \prime}-\psi_{\epsilon}+r c_{\epsilon} \frac{A_{\epsilon}^{r-1}}{\left(T\left[A_{\epsilon}\right]\right)^{s}} \phi_{\epsilon}-s c_{\epsilon} \frac{A_{\epsilon}^{r}}{\left(T\left[A_{\epsilon}\right]\right)^{s+1}} \psi_{\epsilon}=\tau \lambda_{\epsilon} \psi_{\epsilon} .
$$

Here $\lambda_{\epsilon}$ is some complex number, $A_{\epsilon}=w_{\epsilon, \mathbf{t}^{\epsilon}}+\phi_{\epsilon, \mathbf{t}^{\epsilon}}$ with $\mathbf{t}^{\epsilon}$ determined in Section 6 .

In this section, we study the large eigenvalues, i.e., we assume that there exists $c>0$ with $\left|\lambda_{\epsilon}\right| \geq c>0$ for $\epsilon$ small. If $\operatorname{Re}\left(\lambda_{\epsilon}\right) \leq-c$, we are done.(Since then $\lambda_{\epsilon}$ is a stable large eigenvalue.) Therefore we may also assume that $\operatorname{Re}\left(\lambda_{\epsilon}\right) \geq-c$.

We first present the analysis of (8.1), (8.2) for the case $\tau=0$. At the end, we shall explain how we proceed if $\tau>0$ and is small.

By (8.2) we have

$$
\psi_{\epsilon}=T^{\prime}\left[A_{\epsilon}\right]\left(\phi_{\epsilon}\right) .
$$

First of all, since we are concerned only with those eigenvalues such that $\operatorname{Re}\left(\lambda_{\epsilon}\right) \geq-c$, we see that by following the same argument as in the proof as (2) of Theorem 3.2, we have that $\left|\lambda_{\epsilon}\right| \leq C$ for some positive constant $C$ (independent of $\epsilon>0$ ).

Recall the definition of $\phi_{\epsilon, j}$ given in (5.7).

From (8.1) and the facts that $\operatorname{Re}\left(\lambda_{\epsilon}\right) \geq-c$ and that $w_{\epsilon, \mathbf{t}^{\epsilon}}$ has exponential decay, we have that

$$
\phi_{\epsilon}=\sum_{j=1}^{K} \phi_{\epsilon, j}+\text { e.s.t. }
$$

Then we extend $\phi_{\epsilon, j}$ to a function defined on $R^{1}$ such that

$$
\left\|\phi_{\epsilon, j}\right\|_{H^{1}\left(R^{1}\right)} \leq C\left\|\phi_{\epsilon, j}\right\|_{H^{1}\left(\Omega_{\epsilon}\right)}, \quad j=1, \ldots, K .
$$

Without loss of generality we may assume that $\left\|\phi_{\epsilon}\right\|_{\epsilon}=\left\|\phi_{\epsilon}\right\|_{H^{1}\left(\Omega_{\epsilon}\right)}=1$. Then $\left\|\phi_{\epsilon, j}\right\|_{\epsilon} \leq C$. By taking a subsequence of $\epsilon$, we may also assume that $\phi_{\epsilon, j} \rightarrow \phi_{j}$ as $\epsilon \rightarrow 0$ in $H^{1}(R)$ for $j=1, \ldots, K$.

Sending $\epsilon \rightarrow 0$ with $\lambda_{\epsilon} \rightarrow \lambda_{0}$, this implies (as in Section 5)

$$
\begin{gathered}
L \Phi=\Delta \Phi-\Phi+p w^{p-1} \Phi \\
-q r(I+s \mathcal{B})^{-1} \mathcal{B}\left(\int_{R} w^{r-1} \mathcal{B} \Phi\right)\left(\int_{R} w^{r}\right)^{-1} w^{p}=\lambda_{0} \Phi,
\end{gathered}
$$

where

$$
\Phi=\left(\begin{array}{l}
\phi_{1} \\
\phi_{2} \\
\vdots \\
\phi_{N}
\end{array}\right) \in\left(H^{2}(R)\right)^{N}
$$


Then we have

TheOREM 8.1. Let $\lambda_{\epsilon}$ be an eigenvalue of (8.1) and (8.2) such that $\operatorname{Re}\left(\lambda_{\epsilon}\right)>-c$ for some $c>0$.

(1) Suppose that (for suitable sequences $\epsilon_{n} \rightarrow 0$ ) we have $\lambda_{\epsilon_{n}} \rightarrow \lambda_{0} \neq 0$. Then $\lambda_{0}$ is an eigenvalue of the problem (NLEP) given in (8.4).

(2) Let $\lambda_{0} \neq 0$ with $\operatorname{Re}\left(\lambda_{0}\right)>0$ be an eigenvalue of the problem (NLEP) given in (8.4). Then for $\epsilon$ sufficiently small, there is an eigenvalue $\lambda_{\epsilon}$ of (8.1) and (8.2) with $\lambda_{\epsilon} \rightarrow \lambda_{0}$ as $\epsilon \rightarrow 0$.

Proof. (1) of Theorem 8.1 follows by asymptotic analysis similar to Section 5 .

To prove (2) of Theorem 8.1, we follow the argument given in Section 2 of [5], where the following eigenvalue problem was studied:

$$
\left\{\begin{array}{l}
\epsilon^{2} \Delta h-h+p u_{\epsilon}^{p-1} h-\frac{q r}{s+1+\tau \lambda_{\epsilon}} \frac{\int_{\Omega} u_{\epsilon}^{r-1} h}{\int_{\Omega} u_{\epsilon}^{r}} u_{\epsilon}^{p}=\lambda_{\epsilon} h \text { in } \Omega, \\
h=0 \text { on } \partial \Omega
\end{array}\right.
$$

where $u_{\epsilon}$ is a solution of the single equation

$$
\left\{\begin{array}{l}
\epsilon^{2} \Delta u_{\epsilon}-u_{\epsilon}+u_{\epsilon}^{p}=0 \text { in } \Omega \\
u_{\epsilon}>0 \text { in } \Omega, u_{\epsilon}=0 \text { on } \partial \Omega .
\end{array}\right.
$$

Here $1<p<\frac{n+2}{n-2}$ if $n \geq 3$ and $1<p<+\infty$ if $n=1,2, \frac{q r}{(s+1)(p-1)}>1$ and $\Omega \subset R^{n}$ is a smooth bounded domain. If $u_{\epsilon}$ is a single interior peak solution, then it can be shown ([35]) that the limiting eigenvalue problem is a NLEP

$$
\Delta \phi-\phi+p w^{p-1} \phi-\frac{q r}{s+1+\tau \lambda_{0}} \frac{\int_{R^{N}} w^{r-1} \phi}{\int_{R^{N}} w^{r}} w^{p}=\lambda_{0} \phi
$$

where $w$ is the corresponding ground state solution in $R^{n}$ :

$$
\Delta w-w+w^{p}=0, w>0 \text { in } R^{n}, w=w(|y|) \in H^{1}\left(R^{n}\right) .
$$

Dancer in [5] showed that if $\lambda_{0} \neq 0, \operatorname{Re}\left(\lambda_{0}\right)>0$ is an unstable eigenvalue of (8.6), then there exists an eigenvalue $\lambda_{\epsilon}$ of (8.5) such that $\lambda_{\epsilon} \rightarrow \lambda_{0}$.

We now follow his idea. Let $\lambda_{0} \neq 0$ be an eigenvalue of problem (8.4) with $\operatorname{Re}\left(\lambda_{0}\right)>0$. We first note that from the equation for $\psi_{\epsilon}$, we can express $\psi_{\epsilon}$ in terms of $\phi_{\epsilon}$ (as in (8.3)). Now we rewrite (8.1) as follows:

$$
\phi_{\epsilon}=-R_{\epsilon}\left(\lambda_{\epsilon}\right)\left[\frac{p A_{\epsilon}^{p-1} \phi_{\epsilon}}{H_{\epsilon}^{q}}-\frac{q A_{\epsilon}^{p}}{H_{\epsilon}^{q+1}} \psi_{\epsilon}\right],
$$

where $R_{\epsilon}\left(\lambda_{\epsilon}\right)$ is the inverse of $-\Delta+\left(1+\lambda_{\epsilon}\right)$ in $H^{2}(R)$ (which exists if $\operatorname{Re}\left(\lambda_{\epsilon}\right)>-1$ or $\left.\operatorname{Im}\left(\lambda_{\epsilon}\right) \neq 0\right)$, and $\psi_{\epsilon}=T_{\epsilon}^{\prime}\left[A_{\epsilon}\right]\left(\phi_{\epsilon}\right)$ is given by (8.2). The important thing is that $R_{\epsilon}\left(\lambda_{\epsilon}\right)$ is a compact operator if $\epsilon$ is sufficiently small. The rest of the argument follows in the same way as in [5]. For the sake of limited space, we omit the details here. $\mathrm{Q}$

We now study the stability of (8.1), (8.2) for large eigenvalues explicitly and prove (2.38) and (2.40) of Theorem 2.3.

Suppose now that we have

$$
\left(\frac{q r}{p-1}-s\right) \min _{\sigma \in \sigma(\mathcal{B})} \sigma<1
$$


by Theorem 3.1 (1), there exists a positive eigenvalue of (8.4) and thus by Theorem 8.1, there exists an eigenvalue $\lambda_{\epsilon}$ of (8.1) and (8.2) such that $\operatorname{Re}\left(\lambda_{\epsilon}\right)>c_{0}$ for some positive number $c_{0}>0$. This implies that $\left(A_{\epsilon}, H_{\epsilon}\right)$ is unstable.

Suppose now that

$$
\left(\frac{q r}{p-1}-s\right) \min _{\sigma \in \sigma(\mathcal{B})} \sigma>1,
$$

and (2.37) is satisfied, then by Theorem 3.1 (2), we know that for any nonzero eigenvalue $\lambda_{0}$ of $L$ we have

$$
\operatorname{Re}\left(\lambda_{0}\right)<c_{0}<0 \quad \text { for some } c_{0}>0 \text {. }
$$

So by Theorem 8.1, for $\epsilon$ small enough all nonzero large eigenvalues of (8.1), (8.2) all have strictly negative real parts. We conclude that in this case all eigenvalues $\lambda_{\epsilon}$ of $(8.1),(8.2)$, for which $\left|\lambda_{\epsilon}\right| \geq c>0$ holds, satisfy $\operatorname{Re}\left(\lambda_{\epsilon}\right) \leq-c<0$ for $\epsilon$ small enough. They are all stable.

Finally we comment that when $\tau \neq 0$ and $\tau$ is small, we use Theorem 3.2 to conclude. In this case, the matrix $\mathcal{B}$ will have to be replaced by a matrix $\mathcal{B}_{\tau \lambda_{\epsilon}}$ which depends on $\tau \lambda_{\epsilon}$. (In fact, one just replaces the Green's function $G_{D}$ by the following Green's function:

$$
\left.D \Delta G-\left(1+\tau \lambda_{\epsilon}\right) G+\delta_{z}=0, \quad G^{\prime}( \pm 1, z)=0\right) .
$$

It is easy to check that the new matrix will have eigenvalues satisfying (3.2). The rest follows in the same way as before.

In conclusion, we have finished the study of large eigenvalues. It remains to study small eigenvalues only.

In the next section we shall study the eigenvalues $\lambda_{\epsilon}$ which tend to zero as $\epsilon \rightarrow 0$.

9. Stability Analysis: Small Eigenvalues. We now study small eigenvalues for (8.1) and (8.2). Namely, we assume that $\lambda_{\epsilon} \rightarrow 0$ as $\epsilon \rightarrow 0$.

Let

$$
\bar{w}_{\epsilon}=w_{\epsilon, \mathbf{t}^{\epsilon}}+\phi_{\epsilon, \mathbf{t}^{\epsilon}}, \quad \bar{H}_{\epsilon}=T\left[w_{\epsilon, \mathbf{t}^{\epsilon}}+\phi_{\epsilon, \mathbf{t}^{\epsilon}}\right],
$$

where $\mathbf{t}^{\epsilon}=\left(t_{1}^{\epsilon}, \ldots, t_{N}^{\epsilon}\right)$.

After scaling, the eigenvalue problem (8.1), (8.2) becomes

$$
\begin{gathered}
\epsilon^{2} \Delta \phi_{\epsilon}-\phi_{\epsilon}+\frac{p \bar{w}_{\epsilon}^{p-1}}{\bar{H}_{\epsilon}^{q}} \phi_{\epsilon}-q \frac{\bar{w}_{\epsilon}^{p}}{\bar{H}_{\epsilon}^{q+1}} \psi_{\epsilon}=\lambda_{\epsilon} \phi_{\epsilon}, \\
D \Delta \psi_{\epsilon}-\psi_{\epsilon}+c_{\epsilon} r \frac{\bar{A}_{\epsilon}^{r-1}}{\bar{H}_{\epsilon}^{s}} \phi_{\epsilon}-s c_{\epsilon} \frac{\bar{A}_{\epsilon}^{r}}{\bar{H}_{\epsilon}^{s+1}} \psi_{\epsilon}=\lambda_{\epsilon} \tau \psi_{\epsilon} .
\end{gathered}
$$

where $c_{\epsilon}$ is given by (4.8).

We take $\tau=0$ for simplicity. As $\tau \lambda_{\epsilon}<<1$ the results in this section are also valid for $\tau$ finite. As we shall prove, the small eigenvalues are of the order $O\left(\epsilon^{2}\right)$. Unlike in the single interior peak case [35], we need to expand the eigenfunction up to the order $O(\epsilon)$ term. (Such an expansion is also needed in the study of boundary spikes for the shadow system (see [4] and [34].)) 
Let us define

$$
\tilde{w}_{\epsilon, j}(x)=\chi\left(\frac{x-t_{j}^{\epsilon}}{r_{0}}\right) \bar{w}_{\epsilon}(x), \quad j=1, \ldots, N,
$$

where $\chi(x)$ and $r_{0}$ are given in (4.3) and (4.4). Similarly as in Section 5, we define

$$
\begin{aligned}
& \mathcal{K}_{\epsilon, \mathbf{t}^{\epsilon}}^{n e w}:=\operatorname{span}\left\{\tilde{w}_{\epsilon, j}^{\prime} \mid j=1, \ldots, N\right\} \subset H^{2}\left(\Omega_{\epsilon}\right), \\
& \mathcal{C}_{\epsilon, \mathbf{t}^{\epsilon}}^{\text {new }}:=\operatorname{span}\left\{\tilde{w}_{\epsilon, j}^{\prime} \mid j=1, \ldots, N\right\} \subset L^{2}\left(\Omega_{\epsilon}\right) .
\end{aligned}
$$

Then it is easy to see that

$$
\bar{w}_{\epsilon}(x)=\sum_{j=1}^{N} \tilde{w}_{\epsilon, j}(x)+\text { e.s.t. }
$$

Note that $\tilde{w}_{\epsilon, j}(x) \sim \hat{\xi}_{j}^{\frac{q}{p-1}} w\left(\frac{x-t_{j}^{\epsilon}}{\epsilon}\right)$ in $H_{l o c}^{2}(-1,1)$ and $\tilde{w}_{\epsilon, j}$ satisfies

$$
\epsilon^{2} \Delta \tilde{w}_{\epsilon, j}-\tilde{w}_{\epsilon, j}+\frac{\left(\tilde{w}_{\epsilon, j}\right)^{p}}{\bar{H}_{\epsilon}^{q}}+\text { e.s.t. }=0
$$

Thus $\tilde{w}_{\epsilon, j}^{\prime}:=\frac{d \tilde{w}_{\epsilon, j}}{d x}$ satisfies

$$
\epsilon^{2} \Delta \tilde{w}_{\epsilon, j}^{\prime}-\tilde{w}_{\epsilon, j}^{\prime}+\frac{p\left(\tilde{w}_{\epsilon, j}\right)^{p-1}}{\left(\bar{H}_{\epsilon}\right)^{q}} \tilde{w}_{\epsilon, j}^{\prime}-q \frac{\tilde{w}_{\epsilon, j}^{p}}{\left(\bar{H}_{\epsilon}\right)^{q+1}} \bar{H}_{\epsilon}^{\prime}+\text { e.s.t. }=0 .
$$

Let us now decompose

$$
\phi_{\epsilon}=\epsilon \sum_{j=1}^{N} a_{j}^{\epsilon} \tilde{w}_{\epsilon, j}^{\prime}+\phi_{\epsilon}^{\perp}
$$

with complex numbers $a_{j}^{\epsilon}$, (the factor $\epsilon$ is for scaling), where

$$
\phi_{\epsilon}^{\perp} \perp \mathcal{K}_{\epsilon, \mathbf{t}^{\epsilon}}^{n e w} .
$$

Suppose that $\left\|\phi_{\epsilon}\right\|_{H^{2}\left(\Omega_{\epsilon}\right)}=1$. Then $\left|a_{j}^{\epsilon}\right| \leq C$.

Similarly, we can decompose

$$
\psi_{\epsilon}=\epsilon \sum_{j=1}^{N} a_{j}^{\epsilon} \psi_{\epsilon, j}+\psi_{\epsilon}^{\perp},
$$

where $\psi_{\epsilon, j}$ satisfies

$$
D \Delta \psi_{\epsilon, j}-\psi_{\epsilon, j}+c_{\epsilon} r \frac{\bar{w}_{\epsilon}^{r-1}}{\bar{H}_{\epsilon}^{s}} \tilde{w}_{\epsilon, j}^{\prime}-s c_{\epsilon} \frac{\bar{w}_{\epsilon}^{r}}{\bar{H}_{\epsilon}^{s+1}} \psi_{\epsilon, j}=0
$$

and $\psi_{\epsilon}^{\perp}$ satisfies

$$
D \Delta \psi_{\epsilon}^{\perp}-\psi_{\epsilon}^{\perp}+c_{\epsilon} r \frac{\bar{w}_{\epsilon}^{r-1}}{\bar{H}_{\epsilon}^{s}} \phi_{\epsilon}^{\perp}-s c_{\epsilon} \frac{\bar{w}_{\epsilon}^{r}}{\bar{H}_{\epsilon}^{s+1}} \psi_{\epsilon}^{\perp}=0 .
$$


Both (9.9) and (9.10) are solved with Neumann boundary conditions.

Substituting the decompositions of $\phi_{\epsilon}$ and $\psi_{\epsilon}$ into (9.2) we have

$$
\begin{gathered}
q \epsilon \sum_{j=1}^{N} a_{j}^{\epsilon}\left(\frac{\left(\tilde{w}_{\epsilon, j}\right)^{p}}{\bar{H}_{\epsilon}^{q+1}} \bar{H}_{\epsilon}^{\prime}-\frac{\left(\bar{w}_{\epsilon}\right)^{p}}{\bar{H}_{\epsilon}^{q+1}} \psi_{\epsilon, j}\right) \\
+\epsilon^{2} \Delta \phi_{\epsilon}^{\perp}-\phi_{\epsilon}^{\perp}+\frac{p \bar{w}_{\epsilon}^{p-1}}{\bar{H}_{\epsilon}^{q}} \phi_{\epsilon}^{\perp}-q \frac{\bar{w}_{\epsilon}^{p}}{\bar{H}_{\epsilon}^{q+1}} \psi_{\epsilon}^{\perp}-\lambda_{\epsilon} \phi_{\epsilon}^{\perp}+\text { e.s.t. } \\
=\lambda_{\epsilon}\left(\epsilon \sum_{j=1}^{N} a_{j}^{\epsilon} \tilde{w}_{\epsilon, j}^{\prime}\right) .
\end{gathered}
$$

Let us first compute

$$
\begin{gathered}
I_{4}:=q \epsilon \sum_{j=1}^{N} a_{j}^{\epsilon}\left(\frac{\left(\tilde{w}_{\epsilon, j}\right)^{p}}{\bar{H}_{\epsilon}^{q+1}} \bar{H}_{\epsilon}^{\prime}-\frac{\left(\bar{w}_{\epsilon}\right)^{p}}{\bar{H}_{\epsilon}^{q+1}} \psi_{\epsilon, j}\right) \\
=q \epsilon \sum_{j=1}^{N} a_{j}^{\epsilon}\left(\frac{\left(\tilde{w}_{\epsilon, j}\right)^{p}}{\bar{H}_{\epsilon}^{q+1}}\left(\bar{H}_{\epsilon}^{\prime}-\psi_{\epsilon, j}\right)\right)-q \epsilon \sum_{j=1}^{N} a_{j}^{\epsilon} \sum_{k \neq j} \frac{\left(\tilde{w}_{\epsilon, k}\right)^{p}}{\bar{H}_{\epsilon}^{q+1}} \psi_{\epsilon, j}+\text { e.s.t. } \\
=q \epsilon \sum_{j=1}^{N} a_{j}^{\epsilon} \frac{\left(\tilde{w}_{\epsilon, j}\right)^{p}}{\bar{H}_{\epsilon}^{q+1}}\left[-\psi_{\epsilon, j}+\bar{H}_{\epsilon}^{\prime}\right] \\
-q \sum_{j=1}^{N} \sum_{k \neq j} a_{k}^{\epsilon} \epsilon \psi_{\epsilon, k} \frac{\tilde{w}_{\epsilon, j}^{p}}{\bar{H}_{\epsilon}^{q+1}} .
\end{gathered}
$$

We can rewrite $I_{4}$ as follows

$$
I_{4}=-q \epsilon \sum_{j=1}^{N} \sum_{k=1}^{N} a_{k}^{\epsilon} \frac{\tilde{w}_{\epsilon, j}^{p}}{\bar{H}_{\epsilon}^{q+1}}\left(\psi_{\epsilon, k}-\bar{H}_{\epsilon}^{\prime} \delta_{j k}\right)+\text { e.s.t. }
$$

Let us also put

$$
\tilde{L}_{\epsilon} \phi_{\epsilon}^{\perp}:=\epsilon^{2} \Delta \phi_{\epsilon}^{\perp}-\phi_{\epsilon}^{\perp}+\frac{p \bar{w}_{\epsilon}^{p-1}}{\bar{H}_{\epsilon}^{q}} \phi_{\epsilon}^{\perp}-q \frac{p \bar{w}_{\epsilon}^{p}}{\bar{H}_{\epsilon}^{q+1}} \psi_{\epsilon}^{\perp}
$$

and

$$
\mathbf{a}_{\epsilon}:=\left(a_{1}^{\epsilon}, \ldots, a_{N}^{\epsilon}\right)^{T} .
$$

Multiplying both sides of $(9.11)$ by $\tilde{w}_{\epsilon, l}^{\prime}$ and integrating over $(-1,1)$, we obtain

$$
\text { r.h.s. }=\epsilon \lambda_{\epsilon} \sum_{j=1}^{N} a_{j}^{\epsilon} \int_{-1}^{1} \tilde{w}_{\epsilon, j}^{\prime} \tilde{w}_{\epsilon, l}^{\prime}
$$




$$
=\lambda_{\epsilon} a_{l}^{\epsilon} \hat{\xi}_{l}^{\frac{2 q}{p-1}} \int_{R}\left(w^{\prime}(y)\right)^{2} d y(1+O(\epsilon))
$$

and

$$
\begin{gathered}
\text { l.h.s. }=\left(-q \epsilon \sum_{j=1}^{N} \sum_{k=1}^{N} a_{k}^{\epsilon} \int_{-1}^{1} \frac{\tilde{w}_{\epsilon, j}^{p}}{\bar{H}_{\epsilon}^{q+1}}\left(\psi_{\epsilon, k}-\bar{H}_{\epsilon}^{\prime} \delta_{j k}\right) \tilde{w}_{\epsilon, l}^{\prime}\right. \\
+\int_{-1}^{1} q \frac{\tilde{w}_{\epsilon, l}^{p}}{\bar{H}_{\epsilon}^{q+1}}\left(\bar{H}_{\epsilon}^{\prime} \phi_{\epsilon}^{\perp}\right) \\
\left.-\int_{-1}^{1} q \frac{\tilde{w}_{\epsilon, l}^{p}}{\bar{H}_{\epsilon}^{q+1}}\left(\psi_{\epsilon}^{\perp} w_{\epsilon, l}^{\prime}\right)\right)(1+o(1)) \\
=\left(J_{1, l}+J_{2, l}+J_{3, l}\right)(1+o(1)),
\end{gathered}
$$

where $J_{i, l}, i=1,2,3$ are defined by the last equality.

We define the vectors

$$
\mathbf{J}_{i}=\left(J_{i, 1}, \ldots, J_{i, N}\right)^{T}, i=1,2,3 .
$$

The following is the key lemma.

LEMma 9.1. We have

$$
\mathbf{J}_{1}=c_{1} \epsilon^{2} \mathcal{H}^{\frac{2 q}{p-1}-1}\left[\left(\nabla^{2} \mathcal{G}_{D}-\mathcal{Q}\right) \mathcal{H}^{\frac{q r}{p-1}-s}-s \nabla \mathcal{G}_{D} \mathcal{H}^{\frac{q r}{p-1}-s-1} \mathcal{P}_{1}\left(\nabla \mathcal{G}_{D}\right)^{T} \mathcal{H}^{\frac{q r}{p-1}-s}\right] \mathbf{a}_{\epsilon}+o\left(\epsilon^{2}\right),
$$

$$
\mathbf{J}_{2}=o\left(\epsilon^{2}\right)
$$

and

$$
\begin{gathered}
\mathbf{J}_{3}=c_{1} \epsilon^{2} \mathcal{H}^{\frac{2 q}{p-1}-1}\left[\frac{q r}{p-1} \nabla G_{D} \mathcal{H}^{\frac{q r}{p-1}-s-1} \mathcal{P}_{2}\left(\nabla \mathcal{G}_{D}\right)^{T} \mathcal{H}^{\frac{q r}{p-1}-s}\right. \\
\left.+s \nabla \mathcal{G}_{D} \mathcal{H}^{\frac{q r}{p-1}-s-1} \frac{q r}{p-1} \mathcal{P}_{1} \mathcal{G}_{D} \mathcal{H}^{\frac{q r}{p-1}-s-1} \mathcal{P}_{2}\left(\nabla \mathcal{G}_{D}\right)^{T} \mathcal{H}^{\frac{q r}{p-1}-s}\right] \mathbf{a}_{\epsilon}+o\left(\epsilon^{2}\right),
\end{gathered}
$$

where $c_{1}$ is given by (6.5) and $\mathcal{P}_{1}$ and $\mathcal{P}_{2}$ are defined by (2.18) and (2.19), respectively. Recall that $\mathcal{G}_{D}$ are $\mathcal{H}$ are introduced in (2.2) and (2.9), respectively, and $\mathbf{a}_{\epsilon}$ is given in (9.14).

By Lemma 9.1, Theorem 2.3 can be proved. Indeed, note that

$$
\begin{gathered}
s \nabla \mathcal{G}_{D} \mathcal{H}^{\frac{q r}{p-1}-s-1} \mathcal{P}_{1}\left(\nabla \mathcal{G}_{D}\right)^{T} \mathcal{H}^{\frac{q r}{p-1}-s} \\
-s \nabla \mathcal{G}_{D} \mathcal{H}^{\frac{q r}{p-1}-s-1} \frac{q r}{p-1} \mathcal{P}_{1} \mathcal{G}_{D} \mathcal{H}^{\frac{q r}{p-1}-s-1} \mathcal{P}_{2}\left(\nabla \mathcal{G}_{D}\right)^{T} \mathcal{H}^{\frac{q r}{p-1}-s} \\
=s \nabla \mathcal{G}_{D} \mathcal{H}^{\frac{q r}{p-1}-s-1} \mathcal{P}_{1}\left(I-\frac{q r}{p-1} \mathcal{G}_{D} \mathcal{H}^{\frac{q r}{p-1}-s-1} \mathcal{P}_{2}\right)\left(\nabla \mathcal{G}_{D}\right)^{T} \mathcal{H}^{\frac{q r}{p-1}-s}
\end{gathered}
$$




$$
=s \nabla \mathcal{G}_{D} \mathcal{H}^{\frac{q r}{p-1}-s-1} \mathcal{P}_{2}\left(\nabla \mathcal{G}_{D}\right)^{T} \mathcal{H}^{\frac{q r}{p-1}-s} .
$$

Combining the estimates for $J_{1}, J_{2}$ and $J_{3}$ and using (9.20), we have

$$
\begin{gathered}
\text { l.h.s. }=J_{1}+J_{2}+J_{3}=c_{1} \epsilon^{2} \mathcal{H}^{\frac{2 q}{p-1}-1} \\
\times\left(\left(\nabla^{2} \mathcal{G}_{D}-\mathcal{Q}\right) \mathcal{H}^{\frac{q r}{p-1}-s}+\left(\frac{q r}{p-1}-s\right) \nabla \mathcal{G}_{D} \mathcal{H}^{\frac{q r}{p-1}-s-1} \mathcal{P}_{2}\left(\nabla \mathcal{G}_{D}\right)^{T} \mathcal{H}^{\frac{q r}{p-1}-s}\right) \mathbf{a}_{\epsilon}+o\left(\epsilon^{2}\right) \\
=c_{1} \epsilon^{2} \mathcal{H} \frac{2 q}{p-1} \mathcal{M}\left(\mathbf{t}^{\epsilon}\right) \mathbf{a}_{\epsilon}+o\left(\epsilon^{2}\right) .
\end{gathered}
$$

Comparing with r.h.s. we have

$$
c_{1} \epsilon^{2} \mathcal{H}^{\frac{2 q}{p-1}} \mathcal{M}\left(\mathbf{t}^{\epsilon}\right) \mathbf{a}_{\epsilon}+o\left(\epsilon^{2}\right)=\lambda_{\epsilon} \mathcal{H}^{\frac{2 q}{p-1}} \mathbf{a}_{\epsilon} \int_{R}\left(w^{\prime}(y)\right)^{2} d y(1+O(\epsilon)) .
$$

Equation (9.21) shows that the small eigenvalues $\lambda_{\epsilon}$ of (9.2) are

$$
\lambda_{\epsilon} \sim \epsilon^{2} c_{2} \sigma\left(\mathcal{M}\left(\mathbf{t}^{0}\right)\right),
$$

where $c_{2}=\frac{c_{1}}{\int_{R}\left(w^{\prime}\right)^{2}}<0$. This shows that if all the eigenvalues of $\mathcal{M}\left(\mathbf{t}^{0}\right)$ are positive, then the small eigenvalues are stable. On the other hand, if $\mathcal{M}\left(\mathbf{t}^{0}\right)$ has a negative eigenvalue, then we can construct eigenfunctions and eigenvalues to make the system unstable.

This proves Theorem 2.3.

Lemma 9.1 follows from the following series of lemmas.

We first study the asymptotic behavior of $\psi_{\epsilon, j}$.

LEMma 9.2. We have

$$
\left(\left(\psi_{\epsilon, k}-\bar{H}_{\epsilon}^{\prime} \delta_{k l}\right)\left(t_{l}^{\epsilon}\right)\right)=-\mathcal{H}^{\frac{q r}{p-1}-s} \nabla \mathcal{G}_{D} \mathcal{P}_{1}^{T}+O(\epsilon) .
$$

Proof. Note that for $l \neq k$, we have

$$
\begin{gathered}
\left(\psi_{\epsilon, k}-\bar{H}_{\epsilon}^{\prime} \delta_{k l}\right)\left(t_{l}^{\epsilon}\right)=\psi_{\epsilon, k}\left(t_{l}^{\epsilon}\right) \\
=c_{\epsilon} r \int_{-1}^{1} G_{D}\left(t_{l}^{\epsilon}, z\right) \frac{\bar{w}_{\epsilon}^{r-1}}{\bar{H}_{\epsilon}^{s}} \tilde{w}_{\epsilon, k}^{\prime} d z-c_{\epsilon} s \int_{-1}^{1} G_{D}\left(t_{l}^{\epsilon}, z\right) \frac{\bar{w}_{\epsilon}^{r}}{\bar{H}_{\epsilon}^{s+1}} \psi_{\epsilon, k} d z \\
=-\nabla_{t_{k}^{\epsilon}} G_{D}\left(t_{k}^{\epsilon}, t_{l}^{\epsilon}\right) \hat{\xi}_{k}^{\frac{q r}{p-1}-s}-s \sum_{m=1}^{N} G_{D}\left(t_{l}^{\epsilon}, t_{m}^{\epsilon}\right) \psi_{\epsilon, k}\left(t_{m}^{\epsilon}\right) \hat{\xi}_{m}^{\frac{q r}{p-1}-(s+1)}+O(\epsilon) .
\end{gathered}
$$

Next we compute $\psi_{\epsilon, l}-\bar{H}_{\epsilon}^{\prime}$ near $t_{l}^{\epsilon}$ :

$$
\begin{gathered}
\bar{H}_{\epsilon}(x)=c_{\epsilon} \int_{-1}^{1} G_{D}(x, z) \frac{\bar{w}_{\epsilon}^{r}}{\bar{H}_{\epsilon}^{s}} \\
=c_{\epsilon} \int_{-\infty}^{+\infty} K_{D}(|z|) \frac{\tilde{w}_{\epsilon, l}^{r}}{\bar{H}_{\epsilon}^{s}}(x+z) d z-c_{\epsilon} \int_{-1}^{1} H_{D}(x, z) \frac{\tilde{w}_{\epsilon, l}^{r}}{\bar{H}_{\epsilon}^{s}} d z
\end{gathered}
$$




$$
+c_{\epsilon} \sum_{k \neq l} \int_{-1}^{1} G_{D}(x, z) \frac{\tilde{w}_{\epsilon, k}^{r}}{\bar{H}_{\epsilon}^{s}} .
$$

So

$$
\begin{gathered}
\bar{H}_{\epsilon}^{\prime}=c_{\epsilon} \int_{-\infty}^{+\infty} K_{D}(|z|)\left(r \frac{\tilde{w}_{\epsilon, l}^{r-1}}{\bar{H}_{\epsilon}^{s}}(x+z)\right) d z-c_{\epsilon} \int_{-1}^{1} H_{D}(x, z) r \frac{\tilde{w}_{\epsilon, l}^{r-1}}{\bar{H}_{\epsilon}^{s}} d z \\
+c_{\epsilon} \sum_{k \neq l} \int_{-1}^{1} G_{D}(x, z) r \frac{\tilde{w}_{\epsilon, k}^{r-1}}{\bar{H}_{\epsilon}^{s}} d z \\
-s c_{\epsilon} \int_{-1}^{1} G_{D}(x, z) \frac{\bar{w}_{\epsilon}^{r}}{\bar{H}_{\epsilon}^{s+1}} \bar{H}_{\epsilon}^{\prime} d z .
\end{gathered}
$$

Thus

$$
\begin{gathered}
\bar{H}_{\epsilon}^{\prime}-\psi_{\epsilon, l}=-s c_{\epsilon} \int_{-1}^{1} K_{D}(|x-z|) \frac{\tilde{w}_{\epsilon, l}^{r}}{\bar{H}_{\epsilon}^{s+1}} \bar{H}_{\epsilon}^{\prime} \\
-c_{\epsilon} \int_{-1}^{1} \nabla_{x} H_{D}(x, z) \frac{\tilde{w}_{\epsilon, l}^{r}}{\bar{H}^{s}}+c_{\epsilon} \sum_{k \neq l} \int_{-1}^{1} \nabla_{x} G_{D}(x, z) \frac{\tilde{w}_{\epsilon, k}^{r}}{\bar{H}_{\epsilon}^{s}} \\
-\left(-c_{\epsilon} \int_{-1}^{1} H_{D}(x, z) \frac{r \tilde{w}_{\epsilon, l}^{r}}{\bar{H}^{s}} \tilde{w}_{\epsilon, l}^{\prime}-c_{\epsilon} s \int_{-1}^{1} G_{D}(x, z) \frac{\bar{w}_{\epsilon}^{r}}{\bar{H}_{\epsilon}^{s+1}} \psi_{\epsilon, l}\right) .
\end{gathered}
$$

Therefore we have,

$$
\begin{gathered}
\bar{H}_{\epsilon}^{\prime}\left(t_{l}^{\epsilon}\right)-\psi_{\epsilon, l}\left(t_{l}^{\epsilon}\right)=-c_{\epsilon} \int_{-1}^{1} \nabla_{t_{l}^{\epsilon}} H\left(t_{l}^{\epsilon}, z\right) \frac{\tilde{w}_{\epsilon, l}^{r}}{\bar{H}^{s}}+c_{\epsilon} \sum_{k \neq l} \int_{-1}^{1} \nabla_{t_{l}^{\epsilon}} G\left(t_{l}^{\epsilon}, z\right) \frac{\tilde{w}_{\epsilon, k}^{r}}{\bar{H}_{\epsilon}^{s}} \\
-\nabla_{t_{l}^{\epsilon}} H_{D}\left(t_{l}^{\epsilon}, t_{l}^{\epsilon}\right) \hat{\xi}_{l}^{\frac{q r}{p-1}-s}-s \sum_{k=1}^{N} G_{D}\left(t_{l}^{\epsilon}, t_{k}^{\epsilon}\right) \hat{\xi}_{k}^{\frac{q r}{p-1}-(s+1)} \psi_{\epsilon, l}\left(t_{k}^{\epsilon}\right)+O(\epsilon) \\
=-\nabla_{t_{l}^{\epsilon}} H_{D}\left(t_{l}^{\epsilon}, t_{l}^{\epsilon}\right) \hat{\xi}_{l}^{\frac{q r}{p-1}-s}-s \sum_{k=1}^{N} G_{D}\left(t_{l}^{\epsilon}, t_{k}^{\epsilon}\right) \hat{\xi}_{k}^{\frac{q r}{p-1}-(s+1)} \psi_{\epsilon, l}\left(t_{k}^{\epsilon}\right)+O(\epsilon) .
\end{gathered}
$$

Solving the equations (9.23) and (9.24), we have (9.22).

Similar to Lemma 9.2, we have

LEMMA 9.3. We have

$$
\begin{aligned}
& \left(\psi_{\epsilon, k}-\bar{H}_{\epsilon}^{\prime} \delta_{l k}\right)\left(t_{l}^{\epsilon}+\epsilon y\right)-\left(\psi_{\epsilon, k}-\bar{H}_{\epsilon}^{\prime} \delta_{l k}\right)\left(t_{l}^{\epsilon}\right) \\
& =-\epsilon y\left[\nabla_{t_{l}^{\epsilon}} \nabla_{t_{k}^{\epsilon}} G_{D}\left(t_{l}^{\epsilon}, t_{k}^{\epsilon}\right)-q_{l k} \delta_{l k}\right] \hat{\xi}_{k}^{\frac{q r}{p-1}-s}
\end{aligned}
$$




$$
-\epsilon y s \sum_{m=1}^{N} \nabla_{t_{l}^{\epsilon}} G_{D}\left(t_{l}^{\epsilon}, t_{m}^{\epsilon}\right) \psi_{\epsilon, k}\left(t_{m}^{\epsilon}\right) \hat{\xi}_{m}^{\frac{q r}{p-1}-(s+1)}+O\left(\epsilon^{2} y^{2}\right)
$$

where $q_{l k}$ is defined at (2.16).

We next study the asymptotic expansion of $\phi_{\epsilon}^{\perp}$. Let us first denote

$$
\phi_{\epsilon, j}^{1}=\sum_{l=1}^{N}\left(\frac{q}{p-1} \hat{\xi}^{\frac{q}{p-1}-1} \nabla_{t_{j}^{\epsilon}} \hat{\xi}_{l} \tilde{w}_{\epsilon, l}\right), \quad \phi_{\epsilon}^{1}:=\epsilon \sum_{j=1}^{N} a_{j}^{\epsilon} \phi_{\epsilon, j}^{1} .
$$

Then we have

LEMma 9.4. For $\epsilon$ sufficiently small, we have

$$
\left\|\phi_{\epsilon}^{\perp}-\phi_{\epsilon}^{1}\right\|_{H^{2}(-1 / \epsilon, 1 / \epsilon)}=O\left(\epsilon^{2}\right) .
$$

Proof. Before we prove Lemma 9.4, we first obtain a relation between $\psi_{\epsilon}^{\perp}$ and $\phi_{\epsilon}^{\perp}$. Note that similar to the proof of Proposition 5.1, $\tilde{L}_{\epsilon}$ is invertible from $\left(\mathcal{K}_{\epsilon}^{\text {new }}\right)^{\perp}$ to $\left(\mathcal{C}_{\epsilon}^{\text {new }}\right)^{\perp}$. By Lemma 9.2 and the fact that $\tilde{L}_{\epsilon}$ is invertible, we deduce that

$$
\left\|\phi_{\epsilon}^{\perp}\right\|_{H^{2}\left(-\frac{1}{\epsilon}, \frac{1}{\epsilon}\right)}=O(\epsilon)
$$

Let us decompose

$$
\tilde{\phi}_{\epsilon, j}=\frac{\phi_{\epsilon}^{\perp}}{\epsilon} \chi\left(\frac{x-t_{j}^{\epsilon}}{r_{0}}\right)
$$

Then

$$
\phi_{\epsilon}^{\perp}=\epsilon \sum_{j=1}^{N} \tilde{\phi}_{\epsilon, j}+\text { e.s.t. }
$$

Suppose that

$$
\tilde{\phi}_{\epsilon, j} \rightarrow \phi_{j} \text { in } H^{1}
$$

Let

$$
\Phi_{0}=\left(\phi_{1}, \ldots, \phi_{N}\right)^{T}
$$

Then we have by the equation for $\psi_{\epsilon}^{\perp}$ (similar to the proof of Lemma 9.2):

$$
\begin{gathered}
\psi_{\epsilon}^{\perp}\left(t_{j}^{\epsilon}\right)=\epsilon \sum_{k=1}^{N} c_{\epsilon} r \int_{-1}^{1} G_{D}\left(t_{j}^{\epsilon}, z\right) \frac{\bar{w}_{\epsilon}^{r-1}}{\bar{H}_{\epsilon}^{s}} \tilde{\phi}_{\epsilon, k} d z \\
-c_{\epsilon} s \int_{-1}^{1} G_{D}\left(t_{j}^{\epsilon}, z\right) \frac{\bar{w}_{\epsilon}^{r}}{\bar{H}_{\epsilon}^{s+1}} \psi_{\epsilon}^{\perp} d z+\text { e.s.t. } \\
=-\epsilon r \sum_{k=1}^{N} G_{D}\left(t_{j}^{\epsilon}, t_{k}^{\epsilon}\right) \hat{\xi}_{k}^{\frac{q(r-1)}{p-1}}-s \frac{\int_{R} w^{r-1} \phi_{k}}{\int_{R} w^{r}}
\end{gathered}
$$




$$
-s \sum_{k=1}^{N} G_{D}\left(t_{j}^{\epsilon}, t_{k}^{\epsilon}\right) \psi_{\epsilon}^{\perp}\left(t_{k}^{\epsilon}\right) \hat{\xi}_{k}^{\frac{q r}{p-1}-(s+1)}+O\left(\epsilon^{2}\right)
$$

Hence

$$
\left(\psi_{\epsilon}^{\perp}\left(t_{1}^{\epsilon}\right), \ldots, \psi_{\epsilon}^{\perp}\left(t_{N}^{\epsilon}\right)\right)^{T}=-\epsilon r \mathcal{P}_{1} \mathcal{G}_{D} \mathcal{H}^{\frac{q(r-1)}{p-1}-s} \frac{\int_{R} w^{r-1} \Phi_{0}}{\int_{R} w^{r}}+O\left(\epsilon^{2}\right) .
$$

Substituting (9.32) into (9.11) and using Lemma 8.2, we have that in the limit $\Phi_{0}$ satisfies

$$
\begin{gathered}
\Delta \Phi_{0}-\Phi_{0}+p w^{p-1} \Phi_{0} \\
-q r \mathcal{H}^{\frac{q}{p-1}-1} \mathcal{P}_{1} \mathcal{G}_{D} \mathcal{H}^{\frac{q(r-1)}{p-1}-s} \frac{\int_{R} w^{r-1} \Phi_{0}}{\int_{R} w^{r}} w^{p} \\
+q \mathcal{H}^{\frac{q}{p-1}-1} \mathcal{P}_{1}\left(\nabla \mathcal{G}_{D}\right)^{T} \mathcal{H}^{\frac{q r}{p-1}-s} \mathbf{a}^{0} w^{p}=0
\end{gathered}
$$

where

$$
\mathbf{a}^{0}=\lim _{\epsilon \rightarrow 0} \mathbf{a}^{\epsilon}
$$

So

$$
\begin{gathered}
\Phi_{0}=-\frac{q}{p-1}\left(I-\frac{q r}{p-1} \mathcal{H}^{\frac{q}{p-1}-1} \mathcal{P}_{1} \mathcal{G}_{D} \mathcal{H}^{\frac{q(r-1)}{p-1}-s}\right)^{-1} \mathcal{H}^{\frac{q}{p-1}-s} \mathcal{P}_{1}\left(\nabla \mathcal{G}_{D}\right)^{T} \mathcal{H}^{\frac{q r}{p-1}-s} \mathbf{a}^{0} w \\
=-\frac{q}{p-1} \mathcal{H}^{\frac{q}{p-1}-1}\left(I-\left(\frac{q r}{p-1}-s\right) \mathcal{G}_{D} \mathcal{H}^{\frac{q r}{p-1}-s-1}\right)^{-1}\left(\nabla \mathcal{G}_{D}\right)^{T} \mathcal{H}^{\frac{q r}{p-1}-s} \mathbf{a}^{0} w \\
=-\frac{q}{p-1} \mathcal{H}^{\frac{q}{p-1}-1} \mathcal{P}_{2}\left(\nabla \mathcal{G}_{D}\right)^{T} \mathcal{H}^{\frac{q r}{p-1}-s} \mathbf{a}^{0} w
\end{gathered}
$$

Now we compare $\Phi_{0}$ with $\phi_{\epsilon}^{1}$. By definition

$$
\begin{aligned}
& \phi_{\epsilon}^{1}=\epsilon \sum_{k=1}^{N} a_{k}^{\epsilon} \sum_{m=1}^{N}\left(\frac{q}{p-1} \hat{\xi}_{m}^{\frac{q}{p-1}-1} \nabla_{t_{k}^{\epsilon}} \hat{\xi}_{m} \tilde{w}_{\epsilon, m}\right) \\
& =\epsilon \sum_{m=1}^{N} \frac{q}{p-1} \hat{\xi}_{m}^{\frac{q}{p-1}-1}\left[\sum_{k=1}^{N}\left(\nabla_{t_{k}^{\epsilon}} \hat{\xi}_{m} a_{k}^{\epsilon}\right)\right] \tilde{w}_{\epsilon, m} .
\end{aligned}
$$

On the other hand

$$
\phi_{\epsilon}^{\perp}=\epsilon \sum_{j=1}^{N} \tilde{\phi}_{\epsilon, j}+\text { e.s.t. }
$$




$$
=\epsilon \sum_{j=1}^{N} \phi_{j}\left(\frac{x-t_{j}^{\epsilon}}{\epsilon}\right)+O\left(\epsilon^{2}\right) .
$$

Using (9.33) and (2.15), and comparing (9.34) and (9.35), we obtain (9.27).

From Lemma 9.4, we have that

$$
\left(\psi_{\epsilon}^{\perp}\left(t_{1}^{\epsilon}\right), \ldots, \psi_{\epsilon}^{\perp}\left(t_{N}^{\epsilon}\right)\right)^{T}=\epsilon \frac{q r}{p-1} \mathcal{P}_{1} \mathcal{G}_{D} \mathcal{H}^{\frac{q r}{p-1}-s-1} \mathcal{P}_{2}\left(\nabla \mathcal{G}_{D}\right)^{T} \mathcal{H}^{\frac{q r}{p-1}-s}+O\left(\epsilon^{2}\right)
$$

and

$$
\begin{gathered}
\psi_{\epsilon}^{\perp}\left(t_{j}^{\epsilon}+\epsilon y\right)-\psi_{\epsilon}^{\perp}\left(t_{j}^{\epsilon}\right) \\
=-\epsilon^{2} y r \sum_{k=1}^{N} \nabla_{t_{j}^{\epsilon}} G_{D}\left(t_{j}^{\epsilon}, t_{k}^{\epsilon}\right) \hat{\xi}_{k}^{\frac{q(r-1)}{p-1}-s} \frac{\int_{R} w^{r-1} \phi_{k}}{\int_{R} w^{r}} \\
-s \epsilon y \sum_{k=1}^{N} \nabla_{t_{j}^{\epsilon}} G_{D}\left(t_{j}^{\epsilon}, t_{k}^{\epsilon}\right) \psi_{\epsilon}^{\perp}\left(t_{k}^{\epsilon}\right) \hat{\xi}_{k}^{\frac{q r}{p-1}-(s+1)}+O\left(\epsilon^{3} y^{2}\right) .
\end{gathered}
$$

Finally we prove the key lemma - Lemma 9.1.

Proof of Lemma 9.1. The computation of $J_{2}$ follows from Lemma 9.3: In fact, since $\bar{H}_{\epsilon}^{\prime}=o(1)$,

$$
\begin{gathered}
J_{2, l}=-q \epsilon \sum_{k=1}^{N} a_{k}^{\epsilon} \int_{-1}^{1} \frac{\tilde{w}_{\epsilon, l}^{p}}{\bar{H}_{\epsilon}^{q+1}}\left(\psi_{\epsilon, k}-\bar{H}_{\epsilon}^{\prime} \delta_{l k}\right) \tilde{w}_{\epsilon, l}^{\prime}+\text { e.s.t. } \\
=-q \epsilon \sum_{k=1}^{N} a_{k}^{\epsilon} \int_{-1}^{1} \frac{\tilde{w}_{\epsilon, l}^{p}}{\bar{H}_{\epsilon}^{q+1}}\left(\left[\psi_{\epsilon, k}(x)-\bar{H}_{\epsilon}^{\prime}(x) \delta_{l k}\right]-\left[\psi_{\epsilon, k}\left(t_{l}^{\epsilon}\right)-\bar{H}_{\epsilon}^{\prime}\left(t_{l}^{\epsilon}\right) \delta_{l k}\right]\right) \tilde{w}_{\epsilon, l}^{\prime}+o\left(\epsilon^{2}\right) \\
=q \epsilon^{2} \int_{R}\left(y w^{p} w^{\prime}(y)\right) d y \times \hat{\xi}_{l}^{\frac{2 q}{p-1}-1} \\
\sum_{k=1}^{N}\left[\nabla_{t_{l}^{\epsilon}} \nabla_{t_{k}^{\epsilon}} G_{D}\left(t_{l}^{\epsilon}, t_{k}^{\epsilon}\right) \hat{\xi}_{k}^{\frac{q r}{p-1}-s}+s \sum_{m=1}^{N} \nabla_{t_{l}^{\epsilon}} G_{D}\left(t_{l}^{\epsilon}, t_{m}^{\epsilon}\right) \psi_{\epsilon, k}\left(t_{m}^{\epsilon}\right) \hat{\xi}_{m}^{\frac{q r}{p-1}-(s+1)}\right] a_{k}^{\epsilon}+o\left(\epsilon^{2}\right)
\end{gathered}
$$

which, by Lemma 9.2, proves (9.17).

(9.18) follows from Lemma 9.4 and the fact that at $t_{j}^{\epsilon}$

$$
\bar{H}_{\epsilon}\left(t_{j}^{\epsilon}\right)=\hat{\xi}_{j}+O\left(\epsilon^{2}\right), \quad \bar{H}_{\epsilon}^{\prime}\left(t_{j}^{\epsilon}+\epsilon y\right)-\bar{H}_{\epsilon}^{\prime}\left(t_{j}^{\epsilon}\right)=\epsilon \times \text { odd function }+O\left(\epsilon^{2}\right) .
$$

It remains to prove (9.19):

$$
J_{3}=-\int_{-1}^{1} q \frac{\tilde{w}_{\epsilon, l}^{p}}{\bar{H}_{\epsilon}^{q+1}}\left(\psi_{\epsilon}^{\perp} w_{\epsilon, l}^{\prime}\right)
$$




$$
\begin{gathered}
=-\int_{-1}^{1} q \frac{\tilde{w}_{\epsilon, l}^{p}}{\bar{H}_{\epsilon}^{q+1}}\left(\psi_{\epsilon}^{\perp}\left(t_{l}^{\epsilon}\right) w_{\epsilon, l}^{\prime}\right) \\
-\int_{-1}^{1} q \frac{\tilde{w}_{\epsilon, l}^{p}}{\bar{H}_{\epsilon}^{q+1}}\left(\psi_{\epsilon}^{\perp}(x)-\psi_{\epsilon}^{\perp}\left(t_{l}^{\epsilon}\right)\right) w_{\epsilon, l}^{\prime} \\
=-\int_{-1}^{1} q \frac{\tilde{w}_{\epsilon, l}^{p}}{\bar{H}_{\epsilon}^{q+1}}\left(\psi_{\epsilon}^{\perp}(x)-\psi_{\epsilon}^{\perp}\left(t_{l}^{\epsilon}\right)\right) w_{\epsilon, l}^{\prime}+o\left(\epsilon^{2}\right) .
\end{gathered}
$$

Now (9.19) follows from (9.33), (9.36) and (9.37).

10. Appendix A: Computation of the Eigenvalues of $\mathcal{B}$ and $\mathcal{M}$ and the proof of Theorem 2.4. In this appendix, we give a sketch of the computations of the eigenvalues of $\mathcal{B}$ and $\mathcal{M}$ in the case of symmetric $N$-peaked solutions. Then Theorem 2.4 follows from Theorem 2.3. For more detailed computations, we refer the reader to [15] and [28].

We need to consider the three matrices $\mathcal{G}_{D}, \nabla \mathcal{G}_{D}$ and $\nabla^{2} \mathcal{G}_{D}$.

Recall that

$$
t_{j}^{0}=-1+\frac{2 j-1}{N}, \quad j=1, \ldots, N, \quad \theta=\frac{1}{\sqrt{D}} .
$$

By definition, it is easy to compute

$$
\mathcal{G}_{D}=\frac{\theta}{\sinh (2 \theta)}\left(a_{i j}\right), \nabla \mathcal{G}_{D}=\frac{\theta^{2}}{\sinh (2 \theta)}\left(b_{i j}\right), \nabla^{2} \mathcal{G}_{D}=\frac{\theta^{3}}{\sinh (2 \theta)}\left(c_{i j}\right),
$$

where

$$
\begin{gathered}
a_{i j}=\left\{\begin{array}{l}
\cosh \left(\theta\left(1+t_{i}^{0}\right)\right) \cosh \left(\theta\left(1-t_{j}^{0}\right)\right), \text { if } i \leq j ; \\
\cosh \left(\theta\left(1-t_{i}^{0}\right)\right) \cosh \left(\theta\left(1+t_{j}^{0}\right)\right), \text { if } i>j,
\end{array}\right. \\
b_{i j}=\left\{\begin{array}{l}
\sinh \left(\theta\left(1+t_{i}^{0}\right)\right) \cosh \left(\theta\left(1-t_{j}^{0}\right)\right), \text { if } i<j ; \\
\frac{1}{2} \sinh \left(2 \theta t_{i}^{0}\right), \text { if } i=j ; \\
-\sinh \left(\theta\left(1-t_{i}^{0}\right)\right) \cosh \left(\theta\left(1+t_{j}^{0}\right)\right), \text { if } i>j,
\end{array}\right.
\end{gathered}
$$

and

$$
c_{i j}=\left\{\begin{array}{l}
-\sinh \left(\theta\left(1+t_{i}^{0}\right)\right) \sinh \left(\theta\left(1-t_{j}^{0}\right)\right), \text { if } i<j ; \\
-\sinh \left(\theta\left(1+t_{i}^{0}\right)\right) \sinh \left(\theta\left(1-t_{i}^{0}\right)\right)+\frac{1}{2} \sinh (2 \theta), \text { if } i=j ; \\
-\sinh \left(\theta\left(1-t_{i}^{0}\right)\right) \sinh \left(\theta\left(1+t_{j}^{0}\right)\right), \text { if } i>j,
\end{array}\right.
$$

In the symmetric $N$-peaked case, $\hat{\xi}_{1}^{0}=\hat{\xi}_{2}^{0}=\ldots=\hat{\xi}_{N}^{0}=\hat{\xi}_{0}$. Hence

$$
\mathcal{H}=\hat{\xi}_{0} I \text {. }
$$

One can compute $\hat{\xi}_{0}$ explicitly

$$
\hat{\xi}_{0}^{\frac{q r}{p-1}-s-1}=2 \frac{\tanh \left(\frac{\theta}{N}\right)}{\theta} .
$$


Hence

$$
\mathcal{Q}=\left(\frac{\theta^{3}}{2}-\frac{\theta^{3}}{2 \tanh \left(\frac{\theta}{N}\right)}\right) I .
$$

The following three observations make the computation easier:

Observation I: $\mathcal{G}_{D}^{-1}$ is a tridiagonal matrix. More precisely, we have

$$
\mathcal{G}_{D}^{-1}=\sqrt{D}\left(\begin{array}{ccccc}
d_{1} & f_{1} & 0 & \ddots & 0 \\
f_{1} & e_{1} & f_{1} & 0 & \ddots \\
\ddots & \ddots & \ddots & \ddots & \ddots \\
\ddots & 0 & f_{1} & e_{1} & f_{1} \\
0 & \ddots & 0 & f_{1} & d_{1}
\end{array}\right)
$$

where

$$
d_{1}=\operatorname{coth}(2 \theta / N)+\tanh (\theta / N), e_{1}=2 \operatorname{coth}(2 \theta / N), f_{1}=-\operatorname{csch}(2 \theta / N) .
$$

Since $\mathcal{G}_{D}^{-1}$ is a symmetric tridiagonal matrix, we can easily compute the eigenvalues and eigenvectors of $\mathcal{G}_{D}$ as follows:

$$
\begin{gathered}
\lambda_{j}=\theta\left(e_{1}+2 f_{1} \cos \left(\frac{\pi(j-1)}{N}\right)\right)^{-1}, j=1, \ldots, N \\
\mathbf{q}_{1}^{t}=\frac{1}{\sqrt{N}}(1, \ldots, 1), \\
\mathbf{q}_{j}^{t}=\left(q_{1, j}, \ldots, q_{N, j}\right), j=2, \ldots, N, \\
q_{l, j}=\sqrt{\frac{2}{N}}\left(\cos \left(\frac{\pi(j-1)}{N}\left(l-\frac{1}{2}\right)\right), j=2, \ldots, N, l=1, \ldots, N .\right.
\end{gathered}
$$

In summary, if we take

$$
\mathcal{P}_{1}=\left(\mathbf{q}_{1}, \ldots, \mathbf{q}_{N}\right)
$$

then we have

$$
\mathcal{P}_{1}^{-1} \mathcal{G}_{D} \mathcal{P}_{1}=\left(\begin{array}{ccccc}
\lambda_{1} & 0 & 0 & \ddots & 0 \\
0 & \lambda_{2} & 0 & \ddots & 0 \\
\ddots & 0 & \lambda_{j} & 0 & \ddots \\
\ddots & \ddots & \ddots & \ddots & \ddots \\
0 & \ddots & 0 & 0 & \lambda_{N}
\end{array}\right)
$$

Observation II: $\left(\nabla^{2} \mathcal{G}_{D}-\frac{\theta^{3}}{2} I\right)^{-1}$ is a tridiagonal matrix. That is 


$$
\left(\nabla^{2} \mathcal{G}_{D}-\frac{\theta^{3}}{2} I\right)^{-1}=-\theta^{-3}\left(\begin{array}{ccccc}
d_{2} & f_{1} & 0 & \ddots & 0 \\
f_{1} & e_{1} & f_{1} & \ddots & 0 \\
\ddots & f_{1} & e_{1} & f_{1} & \ddots \\
\ddots & \ddots & \ddots & \ddots & \ddots \\
0 & \ddots & 0 & f_{1} & d_{2}
\end{array}\right)
$$

where

$$
d_{2}=\operatorname{coth}(2 \theta / N)+\operatorname{coth}(\theta / N)
$$

Since $\nabla^{2} \mathcal{G}_{D}-\frac{\theta^{3}}{2} I$ is a symmetric tridiagonal matrix, we can easily compute the eigenvalues and eigenvectors of $\nabla^{2} \mathcal{G}_{D}$ as follows:

$$
\begin{gathered}
\mu_{j}=\frac{\theta^{3}}{2}-\theta^{3}\left(e_{1}+2 f_{1} \cos \left(\frac{\pi(j-1)}{N}\right)\right)^{-1}, j=2, \ldots, N, \\
\mu_{1}=\frac{\theta^{3}}{2}-\theta^{3}\left(e_{1}-2 f_{1}\right)^{-1}, \\
\mathbf{v}_{1}^{t}=\frac{1}{\sqrt{N}}\left(1,-1,1, \ldots,(-1)^{N+1}\right), \\
\mathbf{v}_{j}^{t}=\left(v_{1, j}, \ldots, v_{N, j}\right), j=2, \ldots, N, \\
v_{l, j}=\sqrt{\frac{2}{N}}\left(\sin \left(\frac{\pi(j-1)}{N}\left(l-\frac{1}{2}\right)\right), j=2, \ldots, N, l=1, \ldots, N .\right.
\end{gathered}
$$

Thus, if we take

$$
\mathcal{P}_{2}=\left(\mathbf{v}_{1}, \ldots, \mathbf{v}_{N}\right)
$$

then we have

$$
\mathcal{P}_{2}^{-1} \nabla^{2} \mathcal{G}_{D} \mathcal{P}_{2}=\left(\begin{array}{ccccc}
\mu_{1} & 0 & 0 & \ddots & 0 \\
0 & \mu_{2} & 0 & \ddots & 0 \\
\ddots & 0 & \mu_{j} & 0 & \ddots \\
\ddots & \ddots & \ddots & \ddots & \ddots \\
0 & \ddots & 0 & 0 & \mu_{N}
\end{array}\right)
$$

The last observation makes the connection between $\nabla \mathcal{G}_{D}$ and the other two matrices $\mathcal{G}_{D}$ and $\nabla^{2} \mathcal{G}_{D}$. 


\section{Observation III:}

$$
\mathcal{P}_{2}^{-1} \nabla \mathcal{G}_{D} \mathcal{P}_{1}=\left(\begin{array}{ccccc}
\nu_{1} & 0 & 0 & \ddots & 0 \\
0 & \nu_{2} & 0 & \ddots & 0 \\
\ddots & 0 & \nu_{j} & 0 & \ddots \\
\ddots & \ddots & \ddots & \ddots & \ddots \\
0 & \ddots & 0 & 0 & \nu_{N}
\end{array}\right)
$$

where

$$
\nu_{j}=\operatorname{csch}(2 \theta / N) \sin (\pi(j-1) / N) \lambda_{j}, \quad j=1, \ldots, N .
$$

Now let

$$
\tilde{s}=s-\frac{q r}{p-1}, \tilde{\gamma}=\hat{\xi}_{0}^{-\tilde{s}-1} .
$$

Then by (10.8), (10.11) and (10.12), we have that the eigenvalues of $\mathcal{M}$ are given by

$$
m_{j}=\mu_{j}-q_{0}-\tilde{s} \tilde{\gamma} \nu_{j}^{2}\left(1+\tilde{s} \tilde{\gamma} \lambda_{j}\right)^{-1}, \quad j=1, \ldots, N,
$$

where $\mu_{j}$ is given in (10.10), $\nu_{j}$ is given in (10.13), and

$$
q_{0}=\frac{\theta^{3}}{2}-\frac{\theta^{3}}{2 \tanh \left(\frac{\theta}{N}\right)} .
$$

For stability, we need

$$
-\tilde{s} \tilde{\gamma} \min _{j=1, \ldots, N} \lambda_{j}>1
$$

and

$$
\min _{j=1, \ldots, N} m_{j}>0
$$

The first condition (10.14) gives us the following criterion (see [15]):

$$
D<D_{N}^{1} \equiv \frac{1}{\theta_{N, 1}^{2}}, \theta_{N, 1} \equiv \frac{N}{2} \log \left[a+\sqrt{a^{2}-1}\right],
$$

where $a=1+\left[1+\cos \left(\frac{\pi}{N}\right)\right]\left(\frac{q r}{p-1}-s-1\right)^{-1}$.

The second condition (10.15) gives us another critical threshold (see [15]):

$$
D<D_{N}^{2} \equiv \frac{1}{\theta_{N, 2}^{2}}, \theta_{N, 2} \equiv N \log [\sqrt{\beta}+\sqrt{\beta+1}],
$$

where $\beta \equiv\left(\frac{q r}{p-1}-(1+s)\right)^{-1}$.

It is easy to see that $D_{N}^{1}>D_{N}^{2}$. Thus we obtain the stability of symmetric $N$-peaked solution for $D<D_{N} \equiv D_{N}^{2}$ and instability of symmetric $N$-peaked solutions for $D>D_{N}$. (Note that the estimates for small eigenvalues involve no $\tau$.)

This proves Theorem 2.4. 


\section{REFERENCES}

[1] P.W. Bates And P.C. Fife, The dynamics of nucleation for the Cahn-Hilliard equation, SIAM J. Appl. Math., 53 (1993), pp. 990-1008.

[2] P. Bates And G. Fusco, Equilibria with many nuclei for the Cahn-Hilliard equation, J. Differential Equations, 162 (2000), pp. 283-356.

[3] P. Bates, E.N. Dancer AND J. Shi, Multi-spike stationary solutions of the Cahn-Hilliard equation in higher-dimension and instability, Adv. Differential Equations, 4 (1999), pp. 1-69.

[4] P. Bates AND J. ShI, Existence and instability of spike layer solutions to singular perturbation problems, J. Funct. Anal., 196 (2002), pp. 211-264.

[5] E.N. Dancer, On stability and Hopf bifurcations for chemotaxis systems, Methods Appl. Anal., 8 (2001), pp. 245-256.

[6] A. Doelman, R. A. Gardner, and T. J. Kaper, Stability analysis of singular patterns in the $1 D$ Gray-Scott model: a matched asymptotics approach, Phys. D, 122 (1998), pp. 1-36.

[7] A. Doelman, R. A. Gardner, And T. J. KaPer, Large stable pulse solutions in reaction-diffusion equations, Indiana Univ. Math. J., 49 (4) (2000).

[8] A. Doelman, T.J. Kaper, And H. van Der Ploeg, Spatially periodic and aperiodic multi-pulse patterns in the one-dimensional Gierer-Meinhardt equation, Methods Appl. Anal., 8 (2001), pp. 387-414.

[9] A. Doelman, T. J. Kaper, and P. A. Zegeling, Pattern formation in the one-dimensional GrayScott model, Nonlinearity, 10 (1997), pp. 523-563.

[10] A. Floer And A. Weinstein, Nonspreading wave packets for the cubic Schrödinger equation with a bounded potential, J. Funct. Anal., 69 (1986), pp. 397-408.

[11] A. Gierer and H. Meinhardt, A theory of biological pattern formation, Kybernetik (Berlin), 12 (1972), pp. 30-39.

[12] C. Gui AND J. WeI, Multiple interior peak solutions for some singular perturbation problems, J. Differential Equations., 158 (1999), pp. 1-27.

[13] C. Gui, J. Wei AND M. Winter, Multiple boundary peak solutions for some singularly perturbed Neumann problems, Ann. Inst. H. Poincaré Anal. Non Linéaire, 17 (2000), pp. 47-82.

[14] D. Iron And J. Rumsey, Stability of asymmetric spike solutions to the Gierer-Meinhardt system, Chaos, 17 (2007), 037105.

[15] D. Iron, M. WARD, AND J. WeI, The stability of spike solutions to the one-dimensional GiererMeinhardt model, Physica D., 50 (2001), pp. 25-62.

[16] H. Meinhardt, Models of biological pattern formation, Academic Press, London, 1982.

[17] H. Meinhardt, The algorithmic beauty of sea shells, Springer, Berlin, Heidelberg, 2nd edition, 1998.

[18] W.-M. NI, Diffusion, cross-diffusion, and their spike-layer steady states, Notices of Amer. Math. Soc., 45 (1998), pp. 9-18.

[19] W.-M. Ni AND I. TAKAGI, On the shape of least energy solution to a semilinear Neumann problem, Comm. Pure Appl. Math., 41 (1991), pp. 819-851.

[20] W.-M. Ni AND I. TAKAGI, Locating the peaks of least energy solutions to a semilinear Neumann problem, Duke Math. J., 70 (1993), pp. 247-281.

[21] W.-M. Ni, I. Takagi And E. Yanagida, Stability of least energy patterns of the shadow system for an activator-inhibitor model, Japan J. Indust. Appl. Math., 18 (2001), pp. 259-272.

[22] Y. Nishiura, Coexistence of infinitely many stable solutions to reaction-diffusion equation in the singular limit, in "Dynamics reported: Expositions in Dynamical Systems", Volume 3, Editors: C. K. R. T. Jones, U. Kirchgraber, Springer Verlag, New York, (1995).

[23] Y.G. ОH, Existence of semi-classical bound states of nonlinear Schrödinger equations with potentials of the class $(V)_{a}$, Comm. PDE, 13 (1988), pp. 1499-1519.

[24] Y.G. Он, On positive multi-bump bound states of nonlinear Schrödinger equations under multiplewell potentials, Comm. Math. Phys., 131 (1990), pp. 223-253.

[25] I. Takagi, Point-condensation for a reaction-diffusion system, J. Diff. Eqns., 61 (1986), pp. 208-249.

[26] A. M. Turing, The chemical basis of morphogenesis, Phil. Trans. Roy. Soc. Lond. B, 237 (1952), pp. $37-72$.

[27] H. van Der Ploeg, Singular pulse patterns in the Gierer-meinhardt equation, PhD thesis.

[28] M.J. WARD AND J. WeI, Asymmetric spike patterns for the one-dimensional Gierer-Meinhardt model: equilibria and stability, Europ. J. Appl. Math., 13 (2002), pp. 283-320.

[29] M. J. WARD AND J. WeI, Hopf bifurcations of spike solutions for the shadow Gierer-Meinhardt model, Europ. J. Appl. Math., 14 (2003), pp. 677-711.

[30] M. J. WARD AND J. WEI, Hopf bifurcations and oscillatory instabilities of spike solutions for the one-dimensional Gierer-Meinhardt model, J. Nonlinear Science, 13 (2003), pp. 209-264.

[31] J. WEI, On the boundary spike layer solutions of singularly perturbed semilinear Neumann problem, J. Diffential Equations, 134 (1997), pp. 104-133. 
[32] J. WEI, On the interior spike layer solutions of singularly perturbed semilinear Neumann problem, Tohoku Math. J., 50 (1998), pp. 159-178.

[33] J. WeI, On the interior spike layer solutions for some singular perturbation problems, Proc. Royal Soc. Edinburgh, Section A (Mathematics), 128 (1998), pp. 849-874.

[34] J. WEI, Uniqueness and critical spectrum of boundary spike solutions, Proc. Royal Soc. Edinburgh, Section A (Mathematics), 131 (2001), pp. 1457-1480.

[35] J. WeI, On single interior spike solutions of Gierer-Meinhardt system: uniqueness and spectrum estimates, Europ. J. Appl. Math., 10 (1999), pp. 353-378.

[36] J. Wei And M. Winter, Stationary solutions for the Cahn-Hilliard equation, Ann. Inst. H. Poincaré Anal. Non Linéaire, 15 (1998), pp. 459-492.

[37] J. Wei And M. Winter, On the two-dimensional Gierer-Meinhardt system with strong coupling, SIAM J. Math. Anal., 30 (1999), pp. 1241-1263.

[38] J. Wei And M. Winter, Spikes for the two-dimensional Gierer-Meinhardt system: The strong coupling case, J. Differential Equations, 178 (2002), pp. 478-518.

[39] J. Wei And M. Winter, Spikes for the two-dimensional Gierer-Meinhardt system: The weak coupling case, J. Nonlinear Science, 6 (2001), pp. 415-458.

[40] J. Wei and L. Zhang, On a nonlocal eigenvalue problem, Ann. Scuola Norm. Sup. Pisa Cl. Sci., 30 (2001), pp. 41-62. 
